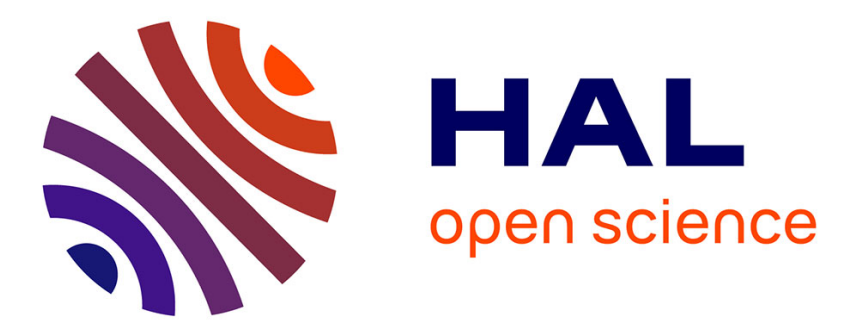

\title{
Nonlocal systems of conservation laws in several space dimensions
}

\author{
Aekta Aggarwal, Rinaldo M. Colombo, Paola Goatin
}

\section{To cite this version:}

Aekta Aggarwal, Rinaldo M. Colombo, Paola Goatin. Nonlocal systems of conservation laws in several space dimensions. SIAM Journal on Numerical Analysis, 2015, 52 (2), pp.963-983. hal-01016784

\section{HAL Id: hal-01016784 \\ https://hal.inria.fr/hal-01016784}

Submitted on 1 Jul 2014

HAL is a multi-disciplinary open access archive for the deposit and dissemination of scientific research documents, whether they are published or not. The documents may come from teaching and research institutions in France or abroad, or from public or private research centers.
L'archive ouverte pluridisciplinaire HAL, est destinée au dépôt et à la diffusion de documents scientifiques de niveau recherche, publiés ou non, émanant des établissements d'enseignement et de recherche français ou étrangers, des laboratoires publics ou privés. 


\title{
NONLOCAL SYSTEMS OF CONSERVATION LAWS IN SEVERAL SPACE DIMENSIONS
}

\author{
AEKTA AGGARWAL*, RINALDO M. COLOMBO ${ }^{\dagger}$, AND PAOLA GOATIN*
}

\begin{abstract}
We present a Lax-Friedrichs type algorithm to numerically integrate a class of nonlocal and nonlinear systems of conservation laws in several space dimensions. The convergence of the approximate solutions is proved, also providing the existence of solution in a slightly more general setting than in other results in the current literature. An application to a crowd dynamics model is considered. This numerical algorithm is then used to test the conjecture that as the convolution kernels converge to a Dirac $\delta$, the nonlocal problem converges to its non-nonlocal analogue.
\end{abstract}

Key words. Nonlocal Conservation Laws, Lax-Friedrichs Scheme.

AMS subject classifications. 35L65, 65M12

1. Introduction. We are concerned with the following class of nonlocal systems of $N$ conservation laws in $d$ space dimensions:

$$
\partial_{t} U+\operatorname{div}_{\mathbf{x}} F(t, \mathbf{x}, U, \eta * U)=0
$$

with a nonlocal flow of the type

$$
\begin{aligned}
& F: \mathbb{R}^{+} \times \mathbb{R}^{n} \times \mathbb{R}^{N} \times \mathbb{R}^{m} \rightarrow \mathbb{R}^{N \times d}
\end{aligned}
$$

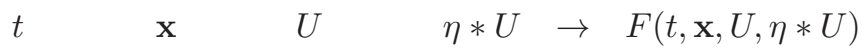

and where the coupling among the equations is due only to the nonlocal terms $\eta * U$, given by, for $\ell=1, \ldots, m$,

$$
(\eta * U)_{\ell}(t, \mathbf{x})=\int_{\mathbb{R}^{n}} \sum_{k=1}^{N} \eta^{\ell, k}(\mathbf{x}-\xi) U^{k}(t, \xi) \mathrm{d} \xi, \quad \text { and } \quad \eta: \mathbb{R}^{n} \rightarrow \mathbb{R}^{m \times N} .
$$

Below we consider a Lax-Friedrichs type algorithm that yields a sequence of approximate solutions to (1.1) that, up to a subsequence, converges to a weak entropy solution of (1.1). As usual, $t$ is time, $\mathbf{x}$ the space variable, $U$ the vector of the unknown densities, $F$ the matrix valued flow and $\eta$ is a matrix of smooth convolution kernels.

Systems of the type (1.1), with the particular coupling considered below, comprise, for instance, sedimentation models [4] and various crowd dynamics models $[8,9,10]$. The nonlocal nature of (1.1) is particularly suitable in describing the behavior of crowds, where each member moves according to her/his evaluation of the crowd density and its variations within her/his horizon. We refer to the several models recently considered in the literature, e.g. [8, 10]. In one space dimension, vehicular traffic offers entirely similar situations, see $[5,9]$. In this connection, we recall that nonlocal systems of conservation laws are widely used in biological applications, e.g., in structured population dynamics, see [19]. The algorithm presented below can easily be adapted to the typical case of the initial boundary value problem for a renewal equation, once the flow at the boundary is pointing into the domain and the source terms are dealt through a standard operator splitting technique, see [18, Chapter 17]. Further applications of nonlocal conservation laws are found in [9] for supply chain models, in [1] for granular material dynamics and the case of a conveyor belt is considered in [13].

\footnotetext{
*Inria Sophia Antipolis-Méditerranée

${ }^{\dagger}$ INDAM Unit, University of Brescia
} 
The key point in the convergence proof lies in obtaining a uniform bound on the total variation of the approximate solutions. However, a bound on the $\mathbf{L}^{\infty}$ norm of the solution is provided below, as well as the $\mathbf{L}^{1}$ Lipschitz continuous dependence of the solution on time. As a consequence, the present result also extends to a wider class of equations the existence part of the results in $[3,4,8,9,10]$. More precisely, in the scalar 1D case, a first convergence proof for a Lax-Friedrichs type algorithm was obtained in [4], see also $[3,5]$. Analytical well posedness results for nonlocal conservation laws in the scalar case were obtained in $[8,9]$ and in $[10]$ in the case of systems. In these references, as in the present work, the actual number of space dimensions is not relevant, if not for the computational cost.

We remark that in the construction below, no divergence free assumption is required, see [7] and the convolution kernel $\eta$ is not required to have compact support.

From an analytical point of view, the limit where $\eta$ converges to a Dirac measure $\delta$ is particularly appealing. Indeed, in that limit system (1.1) tends to a general system of conservation laws in several space dimensions and (at the time of this writing) its well posedness is a well known open problem. Nevertheless, using the algorithm below, in Section 3 we numerically investigate this limit, although the present estimates clearly do not allow to rigorously justify any conclusion in this direction.

The next section presents the main results, while Section 3 is devote to sample numerical integrations of (1.1). The technical details are deferred to Section 4.

2. Main Results. Throughout, we denote $\mathbb{R}_{+}=\left[0,+\infty\left[\right.\right.$ and $\mathbb{R}_{+}^{N}=\left[0,+\infty\left[{ }^{N}\right.\right.$. Aiming at a formal simplification, we consider throughout the case of $d=2$ space dimensions and denote the space variables by $(x, y) \in \mathbb{R}^{2}$. Moreover, we fix our attention to the $k$-th equation in (1.1), for $k=, \ldots, N$, namely

$$
\partial_{t} U^{k}+\partial_{x} f^{k}\left(t, x, y, U^{k}, \eta * U\right)+\partial_{y} g^{k}\left(t, x, y, U^{k}, \vartheta * U\right)=0
$$

where

$$
\begin{aligned}
U(t, x, y) & =\left(U^{1}, \ldots, U^{N}\right)(t, x, y) \\
\eta(x, y) & =\left[\begin{array}{ccc}
\eta^{11} & \ldots & \eta^{1 N} \\
\vdots & \ldots & \vdots \\
\eta^{m 1} & \ldots & \eta^{m N}
\end{array}\right](x, y) \quad \in \mathbb{R}^{N}, \quad \mathbb{R}^{m \times N}, \quad \eta^{\ell, k}(x, y) \quad \in \quad \mathbb{R}, \\
\vartheta(x, y) & =\left[\begin{array}{ccc}
\vartheta^{11} & \ldots & \vartheta^{1 N} \\
\vdots & \ldots & \vdots \\
\vartheta^{m 1} & \ldots & \vartheta^{m N}
\end{array}\right](x, y) \in \mathbb{R}^{m \times N}, \quad \vartheta^{\ell, k}(x, y) \quad \in \mathbb{R},
\end{aligned}
$$

and $(\eta * U)(t, x, y) \in \mathbb{R}^{m},(\vartheta * U)(t, x, y) \in \mathbb{R}^{m}$, where for $\ell=1, \ldots, m$,

$$
\begin{aligned}
& (\eta * U)_{\ell}(t, x, y)=\iint_{\mathbb{R}^{2}} \sum_{k=1}^{N} \eta^{\ell, k}\left(x-x^{\prime}, y-y^{\prime}\right) U^{k}\left(t, x^{\prime}, y^{\prime}\right) \mathrm{d} x^{\prime} \mathrm{d} y^{\prime} \in \mathbb{R}, \\
& (\vartheta * U)_{\ell}(t, x, y)=\iint_{\mathbb{R}^{2}} \sum_{k=1}^{N} \vartheta^{\ell, k}\left(x-x^{\prime}, y-y^{\prime}\right) U^{k}\left(t, x^{\prime}, y^{\prime}\right) \mathrm{d} x^{\prime} \mathrm{d} y^{\prime} \in \mathbb{R} .
\end{aligned}
$$

Throughout, the notation $|\xi|$ is used for $\xi \in \mathbb{R}$, whereas for $\xi \in \mathbb{R}^{\nu},\|\xi\|$ stands for the Euclidean norm. The following assumptions on the maps $f^{k}=f^{k}(t, x, y, u, A), g^{k}=$ $g^{k}(t, x, y, u, B)$ and $\eta, \vartheta$ in $(2.1)$, for $k=1, \ldots, N$ are used below: 
(H0) $f^{k}, g^{k} \in \mathbf{C}^{2}\left(\mathbb{R}^{+} \times \mathbb{R}^{2} \times \mathbb{R} \times \mathbb{R}^{m} ; \mathbb{R}\right) ; \partial_{u} f^{k}, \partial_{u} g^{k} \in \mathbf{L}^{\infty}\left(\mathbb{R}^{+} \times \mathbb{R}^{2} \times \mathbb{R} \times \mathbb{R}^{m} ; \mathbb{R}\right)$; for all $t \in \mathbb{R}^{+},(x, y) \in \mathbb{R}^{2}$ and $A, B \in \mathbb{R}^{m}, f^{k}(t, x, y, 0, A)=g^{k}(t, x, y, 0, B)=0$.

(H1) There exists a constant $M>0$ such that

$$
\begin{array}{r}
\max \left\{\left|\partial_{x} f^{k}(t, x, y, u, A)\right|,\left|\partial_{y} g^{k}(t, x, y, u, B)\right|\right\} \leq M|u|, \\
\max \left\{\left\|\nabla_{A} f^{k}(t, x, y, u, A)\right\|,\left\|\nabla_{B} g^{k}(t, x, y, u, B)\right\|\right\} \leq M|u|, \\
\max \left\{\left|\partial_{x x}^{2} f^{k}(t, x, y, u, A)\right|,\left|\partial_{y y}^{2} g^{k}(t, x, y, u, B)\right|\right\} \leq M|u|, \\
\max \left\{\left|\partial_{x y}^{2} f^{k}(t, x, y, u, A)\right|,\left|\partial_{x y}^{2} g^{k}(t, x, y, u, B)\right|\right\} \leq M|u|, \\
\max \left\{\left\|\partial_{x} \nabla_{A} f^{k}(t, x, y, u, A)\right\|,\left\|\partial_{x} \nabla_{B} g^{k}(t, x, y, u, B)\right\|\right\} \leq M|u|, \\
\max \left\{\left\|\partial_{y} \nabla_{A} f^{k}(t, x, y, u, A)\right\|,\left\|\partial_{y} \nabla_{B} g^{k}(t, x, y, u, B)\right\|\right\} \leq M|u|, \\
\max \left\{\left\|\nabla_{A A}^{2} f^{k}(t, x, y, u, A)\right\|,\left\|\nabla_{B B}^{2} g^{k}(t, x, y, u, B)\right\|\right\} \leq M|u| .
\end{array}
$$

(H2) $\partial_{u} f^{k}, \partial_{u} g^{k} \in \mathbf{W}^{\mathbf{1}, \infty}\left(\mathbb{R}^{+} \times \mathbb{R}^{2} \times \mathbb{R} \times \mathbb{R}^{m} ; \mathbb{R}\right)$.

(H3) $\eta, \vartheta \in\left(\mathbf{C}^{2} \cap \mathbf{W}^{2, \infty}\right)\left(\mathbb{R}^{2} ; \mathbb{R}^{m \times N}\right)$.

REMARK 1. Standard situations of interest lead to compactly supported initial data yielding solutions that are also compactly supported at all finite times. In these cases, it is sufficient that the above uniform boundedness requirements are satisfied on any compact subset of $\mathbb{R}^{2}$. Equivalently, on $f^{k}$ and $g^{k}$ it is enough to assume only conditions (H0) and (H3). The proof is immediate and follows the same lines, for instance, of that in [6, Corollary 2.3].

Recall the definition $[3,4,5,8,9,10]$ of solution to the Cauchy problem for (2.1).

Definition 2.1. Let $U_{o} \in \mathbf{L}^{\infty}\left(\mathbb{R}^{2} ; \mathbb{R}^{N}\right)$. A map $U:[0, T] \rightarrow \mathbf{L}^{\infty}\left(\mathbb{R}^{2} ; \mathbb{R}^{N}\right)$ is a solution to (2.1) with initial datum $U_{o}$ if, for $k=1, \ldots, N$, setting for all $w \in \mathbb{R}$

$$
\begin{aligned}
& \tilde{f}^{k}(t, x, y, w)=f^{k}(t, x, y, w,(\eta * U)(t, x, y)), \\
& \tilde{g}^{k}(t, x, y, w)=g^{k}(t, x, y, w,(\vartheta * U)(t, x, y)),
\end{aligned}
$$

the map $U^{k}$ is a Kružkov solution to the conservation law

$$
\left\{\begin{array}{l}
\partial_{t} U^{k}+\partial_{x} \tilde{f}^{k}\left(t, x, y, U^{k}\right)+\partial_{y} \tilde{g}^{k}\left(t, x, y, U^{k}\right)=0 \\
U^{k}(0, x, y)=U_{o}^{k}(x, y) .
\end{array}\right.
$$

Above, by Kružkov solution we refer to [17, Definition 1]. It is immediate to observe that (H0) ensures that $U(t, x, y) \equiv 0$ solves (2.1) in the sense of Definition 2.1.

Fix a rectangular grid with sizes $\Delta x$ and $\Delta y$ in $\mathbb{R}^{2}$ and choose a time step $\Delta t$. For later use, we also introduce the usual notation

$$
\begin{array}{llll}
t^{n}=n \Delta t, n \in \mathbb{Z} ; & x_{i}=i \Delta x, & x_{i+1 / 2}=\left(i+\frac{1}{2}\right) \Delta x, \quad i \in \mathbb{Z} ; & \lambda_{x}=\Delta t / \Delta x, \\
& y_{j}=j \Delta y, & y_{j+1 / 2}=\left(j+\frac{1}{2}\right) \Delta y, \quad j \in \mathbb{Z} ; & \lambda_{y}=\Delta t / \Delta y .
\end{array}
$$

Throughout, an initial datum $U_{o} \in\left(\mathbf{L}^{\infty} \cap \mathbf{B V}\right)\left(\mathbb{R}^{2} ; \mathbb{R}^{N}\right)$ is fixed and we denote

$$
u_{i j}^{k, 0}=\frac{1}{\Delta x \Delta y} \int_{x_{i-1 / 2}}^{x_{i+1 / 2}} \int_{y_{i-1 / 2}}^{y_{i+1 / 2}} U_{o}^{k}(x, y) \mathrm{d} x \mathrm{~d} y \quad \text { for } i, j \in \mathbb{Z} .
$$


We define a piecewise constant approximate solution $u_{\Delta} \equiv\left(u_{\Delta}^{1}, \ldots, u_{\Delta}^{N}\right)$ to $(2.1)$ by

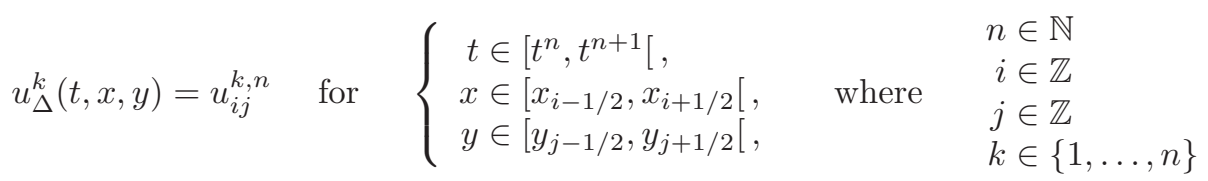

through the 5-points algorithm based on dimensional splitting, see [11, Section 3]:

$$
\begin{aligned}
& u_{i j}^{k, n+1 / 2}=u_{i j}^{k, n} \quad-\lambda_{x}\left[\quad F_{i+1 / 2, j}^{k, n}\left(u_{i j}^{k, n}, u_{i+1, j}^{k, n}\right) \quad-\quad F_{i-1 / 2, j}^{k, n}\left(u_{i-1, j}^{k, n}, u_{i j}^{k, n}\right)\right] \\
& u_{i j}^{k, n}=u_{i j}^{k, n+1 / 2}-\lambda_{y}\left[G_{i, j+1 / 2}^{k, n}\left(u_{i j}^{k, n+1 / 2}, u_{i, j+1}^{k, n+1 / 2}\right)-G_{i, j-1 / 2}^{k, n}\left(u_{i, j-1}^{k, n+1 / 2}, u_{i j}^{k, n+1 / 2}\right)\right]
\end{aligned}
$$

where for fixed $\alpha, \beta$ in $] 0,2 / 3[$, we define

$$
\begin{aligned}
F_{i+1 / 2, j}^{k, n}(u, v) & =\frac{f_{i+1 / 2, j}^{k, n}(u)+f_{i+1 / 2, j}^{k, n}(v)}{2}-\frac{\alpha(v-u)}{2 \lambda_{x}}, \\
G_{i, j+1 / 2}^{k, n}(u, v) & =\frac{g_{i, j+1 / 2}^{k, n}(u)+g_{i, j+1 / 2}^{k, n}(v)}{2}-\frac{\beta(v-u)}{2 \lambda_{y}} \\
f_{i+1 / 2, j}^{k, n}(u) & =f^{k}\left(t^{n}, x_{i+1 / 2}, y_{j}, u, A_{i+1 / 2, j}^{n}\right) \\
g_{i, j+1 / 2}^{k, n}(u) & =g^{k}\left(t^{n}, x_{i}, y_{j+1 / 2}, u, B_{i, j+1 / 2}^{n}\right) .
\end{aligned}
$$

The convolution terms are computed through quadrature formulæ, i.e.,

$$
\begin{aligned}
& A_{i+1 / 2, j}^{n}=\Delta x \Delta y\left(\sum_{l, p \in \mathbb{Z}^{k}} \sum_{k=1}^{N} \eta_{i+1 / 2-l, j-p}^{1, k} u_{l+1 / 2, p}^{k, n}, \ldots, \sum_{l, p \in \mathbb{Z}} \sum_{k=1}^{N} \eta_{i+1 / 2-l, j-p}^{m, k} u_{l+1 / 2, p}^{k, n}\right) \\
& B_{i, j+1 / 2}^{n}=\Delta x \Delta y\left(\sum_{l, p \in \mathbb{Z}} \sum_{k=1}^{N} \vartheta_{i+1 / 2-l, j-p}^{1, k} u_{l+1 / 2, p}^{k, n}, \ldots, \sum_{l, p \in \mathbb{Z}} \sum_{k=1}^{N} \vartheta_{i+1 / 2-l, j-p}^{m, k} u_{l+1 / 2, p}^{k, n}\right),
\end{aligned}
$$

where, for instance, $u_{l+1 / 2, p}^{k, n}$ is a convex combination of $u_{l, p}^{k, n}$ and $u_{l+1, p}^{k, n}$, moreover

$$
\eta_{i+1 / 2, j}^{\ell, k}=\eta^{\ell, k}\left(x_{i+1 / 2}, y_{j}\right) \quad \text { and } \quad \vartheta_{i+1 / 2, j}^{\ell, k}=\vartheta^{\ell, k}\left(x_{i+1 / 2}, y_{j}\right) .
$$

Throughout, we require that $\Delta t$ is chosen in order to satisfy the CFL condition

$$
\lambda_{x} \leq \frac{2 \min \{3 \alpha, 2-3 \alpha\}}{1+6 \max _{k}\left\|\partial_{u} f^{k}\right\|_{\mathbf{L}^{\infty}}}, \quad \lambda_{y} \leq \frac{2 \min \{3 \beta, 2-3 \beta\}}{1+6 \max _{k}\left\|\partial_{u} g^{k}\right\|_{\mathbf{L}^{\infty}}}
$$

and we assume that the mesh sizes are sufficiently small, in the sense that $\Delta x, \Delta y \leq$ $1 /(3 M)$, where $M$ is as in (Ho).

The main result of this paper is the following:

THEOREM 2.2. Let assumptions (HO), (H1), (H3) and conditions (2.6) hold. Fix an initial datum $U_{o} \in\left(\mathbf{L}^{1} \cap \mathbf{L}^{\infty} \cap \mathbf{B V}\right)\left(\mathbb{R}^{2} ; \mathbb{R}_{+}^{N}\right)$. Then, the algorithm (2.3) defines a sequence of approximate solutions which converges, up to a subsequence, to 
a solution $U \in \mathbf{C}^{\mathbf{0}}\left(\mathbb{R}^{+} ; \mathbf{L}^{\mathbf{1}}\left(\mathbb{R}^{2} ; \mathbb{R}_{+}^{N}\right)\right)$ to (2.1) in the sense of Definition 2.1. Moreover, $U$ is locally Lipschitz continuous with respect to the $\mathbf{L}^{\mathbf{1}}$ norm and for all $k \in\{1, \ldots, N\}$ and for all $t \in \mathbb{R}^{+}$, the following bounds hold:

$$
\begin{array}{ll}
\|U(t)\|_{\mathbf{L}^{\infty}\left(\mathbb{R}^{2} ; \mathbb{R}^{N}\right)} \leq e^{\mathcal{C} t}\left\|U_{o}\right\|_{\mathbf{L}^{\infty}\left(\mathbb{R}^{2} ; \mathbb{R}^{N}\right)} & \text { with } \mathcal{C} \text { as in }(4.7), \\
\left\|U^{k}(t)\right\|_{\mathbf{L}^{1}\left(\mathbb{R}^{2} ; \mathbb{R}\right)}=\left\|U_{o}^{k}\right\|_{\mathbf{L}^{1}\left(\mathbb{R}^{2} ; \mathbb{R}\right)}, & \\
\operatorname{TV}\left(U^{k}(t)\right) \leq e^{\mathcal{K}_{1} t} \operatorname{TV}\left(U_{o}^{k}\right)+\mathcal{K}_{2}\left(e^{\mathcal{K}_{1} t}-1\right) & \text { with } \mathcal{K}_{1}, \mathcal{K}_{2} \text { as in }(4.19), \\
\|U(t+\tau)-U(t)\|_{\mathbf{L}^{1}\left(\mathbb{R}^{2} ; \mathbb{R}^{N}\right)} \leq C(t) \tau & \text { with } C(t) \text { as in }(4.22) .
\end{array}
$$

The proof is deferred to Section 4 and is based on the lemmas below. Observe that Theorem 2.2 also ensures the existence of solutions to (2.1) with a flow more general than that considered in $[8,9]$. The solution is proved to be a Lipschitz continuous function of time with respect to the $\mathbf{L}^{\mathbf{1}}$ topology. Moreover, in view of Remark 1, when the initial datum is compactly supported, (H0) and (H3) are sufficient to guarantee the existence of a solution and the bounds above on any finite time interval.

As first step, we prove the positivity of the approximate solutions defined by (2.3).

Lemma 2.3 (Positivity). Let (HO), (H1), (H3) and (2.6) hold. Fix a $U_{o} \in$ $\left(\mathbf{L}^{\mathbf{1}} \cap \mathbf{L}^{\infty} \cap \mathbf{B V}\right)\left(\mathbb{R}^{2} ; \mathbb{R}_{+}^{N}\right)$. Then, the approximate solution $u_{\Delta}$ defined by the algorithm (2.3) satisfies, for all $k \in\{1, \ldots, N\}, t \in \mathbb{R}_{+}$and $(x, y) \in \mathbb{R}^{2}$.

$$
u_{\Delta}^{k}(t, x, y) \geq 0
$$

A consequence of positivity, due to the conservative nature of the algorithm, is the $\mathbf{L}^{1}$ bound.

Lemma 2.4 ( $\mathbf{L}^{\mathbf{1}}$ bound). Let (HO), (H1), (H3) and (2.6) hold. Fix an initial datum $U_{o} \in\left(\mathbf{L}^{\mathbf{1}} \cap \mathbf{L}^{\infty} \cap \mathbf{B V}\right)\left(\mathbb{R}^{2} ; \mathbb{R}_{+}^{N}\right)$. Then, the approximate solution $u_{\Delta}$ defined by the algorithm (2.3) satisfies, for all $k \in\{1, \ldots, N\}$ and $t \in \mathbb{R}_{+}$.

$$
\left\|u_{\Delta}^{k}(t)\right\|_{\mathbf{L}^{1}}=\left\|u_{\Delta}^{k}(0)\right\|_{\mathbf{L}^{1}} .
$$

The proof is omitted, since it is standard, see for instance [3, Lemma 2.4]. Lemma 2.4 allows to prove the $\mathbf{L}^{\infty}$ bound.

Lemma 2.5 ( $\mathbf{L}^{\infty}$ bound). Let (HO), (H1), (H3) and (2.6) hold. Fix an initial datum $U_{o} \in\left(\mathbf{L}^{\mathbf{1}} \cap \mathbf{L}^{\infty} \cap \mathbf{B V}\right)\left(\mathbb{R}^{2} ; \mathbb{R}_{+}^{N}\right)$. Then, there exists a $\mathcal{C}$ depending only on $\eta, \vartheta, f^{1}, \ldots, f^{N}, g^{1}, \ldots, g^{N}$, see $(4.7)$, such that the approximate solution $u_{\Delta}$ defined by the algorithm (2.3) satisfies for all $t \in \mathbb{R}_{+}$,

$$
\left\|u_{\Delta}(t)\right\|_{\mathbf{L}^{\infty}} \leq e^{\mathcal{C} t\left(1+\left\|U_{o}\right\|_{\mathbf{L}^{1}}\right)}\left\|U_{o}\right\|_{\mathbf{L}^{\infty}} .
$$

The previous results allow to get the following key bound on the total variation. Lemma 2.6 (BV bound). Let (HO), (H1), (H2), (H3) and (2.6) hold. Fix an initial datum $U_{o} \in\left(\mathbf{L}^{1} \cap \mathbf{L}^{\infty} \cap \mathbf{B V}\right)\left(\mathbb{R}^{2} ; \mathbb{R}_{+}^{N}\right)$. Then, there exist constants $\mathcal{K}_{1}$ and $\mathcal{K}_{2}$ depending only on $\left\|U_{o}\right\|_{\mathbf{L}^{1}}, \eta, \vartheta, f^{1}, \ldots, f^{N}, g^{1}, \ldots, g^{N}$, see (4.19), such that 
the approximate solution $u_{\Delta}$ defined by the algorithm (2.3) satisfies for all $n$, for all $t \in\left[t^{n}, t^{n+1}[\right.$ and for all $k=1, \ldots, N$

$$
\begin{aligned}
& \sum_{i j}\left(\left|u_{i+1, j}^{k, n}-u_{i j}^{k, n}\right| \Delta y+\left|u_{i, j+1}^{k, n}-u_{i j}^{k, n}\right| \Delta x\right) \\
\leq & e^{\mathcal{K}_{1} t} \sum_{i j}\left(\left|u_{i+1, j}^{k, 0}-u_{i j}^{k, 0}\right| \Delta y+\left|u_{i, j+1}^{k, 0}-u_{i j}^{k, 0}\right| \Delta x\right)+\mathcal{K}_{2}\left(e^{\mathcal{K}_{1} t}-1\right) .
\end{aligned}
$$

Remark that $\mathcal{K}_{2}$ depends on all the components of $U_{o}$. Hence an initial datum with a constant component may well lead to a solution with no constant components.

To prove the discrete entropy inequality, we introduce the following Kružkov numerical entropy flows, see [3, Proposition 2.8], [11, Section 3], [12, Section 4]:

$$
\begin{aligned}
& \Phi_{i+1 / 2, j}^{k, n, \kappa}\left(u_{1}, u_{2}\right)=f_{i+1 / 2, j}^{k, n}\left(u_{1} \vee \kappa, u_{2} \vee \kappa\right)-f_{i+1 / 2, j}^{k, n}\left(u_{1} \wedge \kappa, u_{2} \wedge \kappa\right), \\
& \Gamma_{i, j+1 / 2}^{k, n, \kappa}\left(u_{1}, u_{2}\right)=g_{i, j+1 / 2}^{k, n}\left(u_{1} \vee \kappa, u_{2} \vee \kappa\right)-g_{i, j+1 / 2}^{k, n}\left(u_{1} \wedge \kappa, u_{2} \wedge \kappa\right) .
\end{aligned}
$$

Lemma 2.7 (Discrete entropy condition). Let assumptions (HO), (H1), (H2), (H3) and conditions (2.6) hold. Fix an initial datum $U_{o} \in\left(\mathbf{L}^{\mathbf{1}} \cap \mathbf{L}^{\infty} \cap \mathbf{B V}\right)\left(\mathbb{R}^{2} ; \mathbb{R}_{+}^{N}\right)$. Then, the approximate solution $u_{\Delta}$ defined by the algorithm (2.3) satisfies for all $i, j \in \mathbb{Z}$ and for all $k=1, \ldots, N$ and for all $\kappa \in \mathbb{R}$ the discrete entropy inequality

$$
\begin{aligned}
\left|u_{i j}^{k, n+1}-\kappa\right| & -\left|u_{i j}^{k, n}-\kappa\right|+\lambda_{x}\left(\Phi_{i+1 / 2, j}^{k, n, \kappa}\left(u_{i j}^{k, n}, u_{i+1, j}^{k, n}\right)-\Phi_{i-1 / 2, j}^{k, n, \kappa}\left(u_{i-1, j}^{k, n}, u_{i j}^{k, n}\right)\right) \\
& +\lambda_{x} \operatorname{sgn}\left(u_{i j}^{k, n+1 / 2}-\kappa\right)\left(f_{i+1 / 2, j}^{k, n}(\kappa)-f_{i-1 / 2, j}^{k, n}(\kappa)\right) \\
& +\lambda_{y}\left(\Gamma_{i, j+1 / 2}^{k, n, \kappa}\left(u_{i j}^{k, n+1 / 2}, u_{i, j+1}^{k, n+1 / 2}\right)-\Gamma_{i, j-1 / 2}^{k, n, \kappa}\left(u_{i, j-1}^{k, n+1 / 2}, u_{i j}^{k, n+1 / 2}\right)\right) \\
& +\lambda_{y} \operatorname{sgn}\left(u_{i j}^{k, n+1}-\kappa\right)\left(g_{i, j+1 / 2}^{k, n}(\kappa)-g_{i, j-1 / 2}^{k, n}(\kappa)\right) \quad \leq 0 .
\end{aligned}
$$

The proof is entirely analogous to that of [3, Proposition 2.8].

Lemma 2.8 ( $\mathbf{L}^{\mathbf{1}}$ Lipschitz Continuity in time). Let assumptions (HO), (H1), (H2), (H3) and conditions (2.6) hold. Fix an initial datum $U_{o} \in\left(\mathbf{L}^{1} \cap \mathbf{L}^{\infty} \cap\right.$ $\mathbf{B V})\left(\mathbb{R}^{2} ; \mathbb{R}_{+}^{N}\right)$. Then, for any $\bar{n} \in \mathbb{N}$, there exists a constant $C$ depending on $\left\|U_{o}\right\|_{\mathbf{L}^{1}}$, $\operatorname{TV}\left(U_{o}\right), t^{n}, \lambda_{x}, \lambda_{y} \alpha, \beta$ and on the functions $\eta, \vartheta, f^{k}$ and $g^{k}$, for $k \in\{1, \ldots, N\}$ such that for $n=1, \ldots, \bar{n}$ the approximate solution $u_{\Delta}$ defined by (2.3) satisfies

$$
\left\|u_{\Delta}\left(t^{n+1}\right)-u_{\Delta}\left(t^{n}\right)\right\|_{\mathbf{L}^{1}} \leq C \Delta t
$$

The proof is a straightforward generalization of [3, Lemma 2.7].

\section{Numerical Integrations.}

3.1. A Crowd Dynamics Sample Integration. We now show that the crowd dynamics model introduced in [9] fits in the framework of (2.1). Assume that a group of pedestrians can be described through the density $U=U(t, x, y)$ that satisfies the nonlocal continuity equation

$$
\partial_{t} U+\nabla \cdot(U(1-U)(1-U * \mu) \vec{v})=0 .
$$


Here, the given vector field $\vec{v}=\vec{v}(x, y) \equiv\left(v^{1}(x, y), v^{2}(x, y)\right)$ describes the path followed by the pedestrians. The smooth, non negative and compactly supported function $\mu$ models the way in which each individual averages the density around her/his position to adjust her/his speed.

Lemma 3.1. System (3.1) fits into (2.1) setting

$$
\begin{array}{lll}
N=1, & f(t, x, y, U, A)=U(1-U)(1-A) v^{1}(x, y) \\
m=1, & g(t, x, y, U, B)=U(1-U)(1-B) v^{2}(x, y)
\end{array} \quad \text { and } \quad \begin{array}{ll}
\eta=\mu \\
\vartheta=\mu .
\end{array}
$$

Moreover, if $v \in\left(\mathbf{C}^{2} \cap \mathbf{W}^{2, \infty}\right)\left(\mathbb{R}^{2}, \mathbb{R}^{2}\right)$ and $\mu \in\left(\mathbf{C}^{2} \cap \mathbf{W}^{2, \infty}\right)\left(\mathbb{R}^{2} ; \mathbb{R}\right)$, then Theorem 2.2 applies to any initial datum in $\left(\mathbf{L}^{\mathbf{1}} \cap \mathbf{L}^{\infty} \cap \mathbf{B V}\right)\left(\mathbb{R}^{2} ;[0,1]\right)$.

The proof in Section 4 essentially relies on the invariance of the interval $[0,1]$ for $u$.

We now consider a specific situation. Two groups of people move from different places along a corridor $[0,10] \times[-1,1]$ towards one of its ends and stop in front of $\{9.5\} \times[-1,1]$. Therefore, in $(3.1)$ we choose:

$$
\begin{aligned}
& \vec{v}(x, y)=\left[\begin{array}{c}
\left(1-y^{2}\right)^{3} \exp \left(-1 /(x-9.5)^{2}\right) \chi_{]-\infty, 9.5] \times[-1,1]}(x, y) \\
-2 y \exp \left(1-1 / y^{2}\right) \rho(y)
\end{array}\right] \\
& \tilde{\mu}(x, y)=\left(0.16-x^{2}-y^{2}\right)^{3} \chi_{\left\{(x, y): x^{2}+y^{2} \leq 0.16\right\}}(x, y), \quad \mu(x, y)=\frac{1}{\iint_{\mathbb{R}^{2}} \tilde{\mu}} \tilde{\mu}(x, y),
\end{aligned}
$$

so that $\iint_{\mathbb{R}^{2}} \mu(x, y) \mathrm{d} x \mathrm{~d} y=1$. Here, $\rho \in \mathbf{C}_{\mathbf{c}}^{2}(\mathbb{R} ; \mathbb{R})$ is chosen so that $\rho(y)=1$ for all $y \in[-1,1]$. The role of $\rho$ is to allow conditions (H0), (H1) and (H2) to hold and does
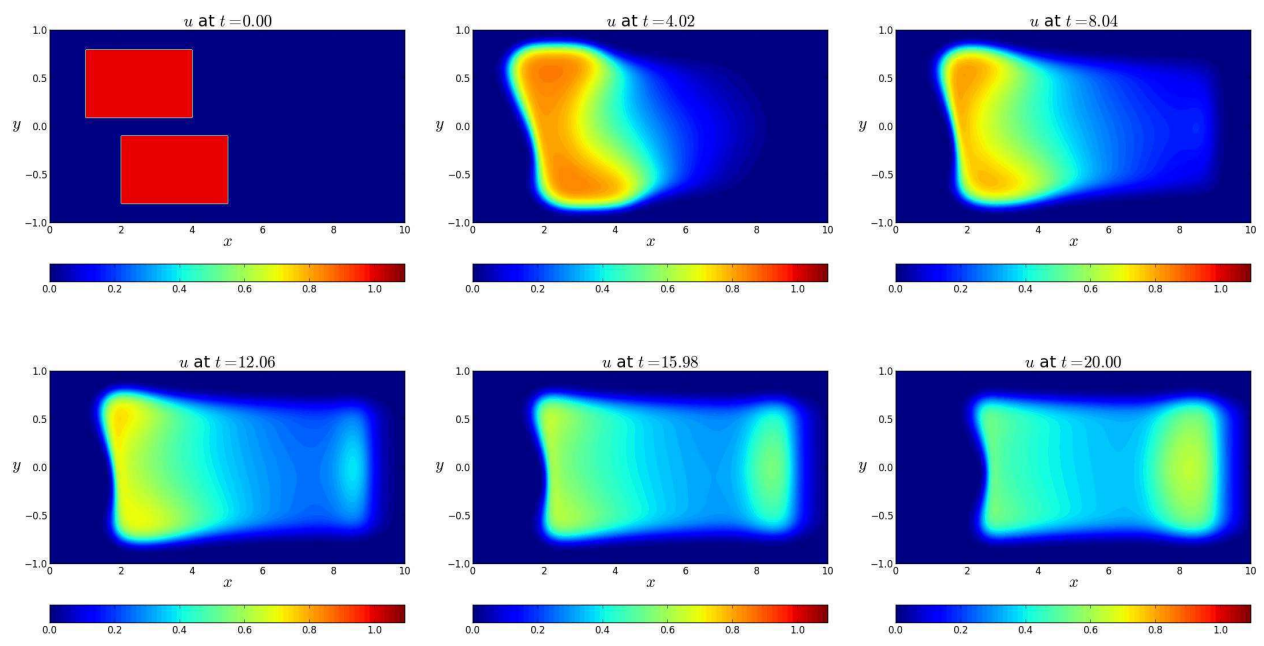

FIG. 3.1. Solution to (3.1)-(3.4) at times $t=0.00,4.02,8.04,12.06,15.98,20.00$ with space mesh $\Delta x=\Delta y=0.0125, \alpha=\beta=0.3333$ in (2.6) and $\Delta t \approx 9.62 E-4$.

not modify the solutions due to the invariance of the space domain $[0,10] \times[-1,1]$. As initial datum we choose:

$$
U_{o}(x, y)=\chi_{[1,4] \times[0.1,0.8]}(x, y)+\chi_{[2,5] \times[-0.8,-0.1]}(x, y) .
$$

The numerical integration of (3.1)-(3.4) obtained with the algorithm described in Section 2 is shown in Figure 3.1. The two groups are seen moving to the right 
and accumulating in front of $x=9.5$. By Definition 2.1, [17, Theorem 3] and the choice (3.4) of the initial datum, the maximal density of solution can not exceed 1 , coherently with the result of the integration. The numerical domain $[0,10] \times[-1,1]$ is chosen invariant with respect to the flow specified in (3.3).

To compute the convergence rate of the scheme (2.3), we apply the algorithm to problem $(3.1)-(3.4)$ on the domain $[0,10] \times[-1,1]$ on the time interval $[0,1]$ with different grid sizes, namely

$$
h=\Delta x=\Delta y=0.05,0.025,0.0125,0.00625 .
$$

and with $\lambda_{x}=\lambda_{y}=0.15$. The convergence rate $\gamma$ is then calculated at time $t=1.0$ by computing the $\mathbf{L}^{\mathbf{1}}$ distance between the numerical solutions $u_{h}$ and $u_{\frac{h}{2}}$ obtained for the grid size $h$ and $\frac{h}{2}$, for each grid size $h$ in (3.5). The results recorded in Table 3.1 show that the numerical scheme $(2.3)$ has a convergence rate $\gamma \approx 0.5$..

\begin{tabular}{|l|c|c||}
\hline \multicolumn{1}{|c|}{$h$} & $\left\|u_{h}-u_{\frac{h}{2}}\right\|_{\mathbf{L}^{1}}$ & $\gamma=\log _{2} \frac{\left\|u_{h}-u_{\frac{h}{2}}\right\|_{\mathbf{L}^{1}}}{\left\|u_{\frac{h}{2}}-u_{\frac{h}{4}}\right\|_{\mathbf{L}^{1}}}$ \\
\hline 0.05 & 0.6565 & 0.2844 \\
\hline 0.025 & 0.5389 & 0.4815 \\
\hline 0.0125 & 0.3860 & 0.5572 \\
\hline 0.00625 & 0.2623 & \\
\hline \multicolumn{2}{|c|}{ TABLE 3.1}
\end{tabular}

Convergence rate $\gamma$ for the numerical scheme $(2.3)$ on the domain $[0,10] \times[-1,1]$ at time $t=1.0$ for the approximate solutions to the problem (3.1)-(3.4) with $\lambda_{x}=\lambda_{y}=0.15$.

3.2. The Nonlocal Keyfitz-Kranzer System. We consider the example of the classical Keyfitz-Kranzer system [15] which is given by

$$
\left\{\begin{array}{l}
\partial_{t} U^{1}+\partial_{x}\left(U^{1} \varphi(U)\right)=0 \\
\partial_{t} U^{2}+\partial_{x}\left(U^{2} \varphi(U)\right)=0
\end{array}\right.
$$

is a $2 \times 2$ system of conservation laws in one space dimension in the unknown $U=$ $\left(U^{1}, U^{2}\right)$. System (3.6) has been extended and studied from many different points of view. For instance, the existence of solutions in the case of several space dimensions was obtained in [2, Theorem 2.6]. The convergence of approximate solutions was proved in [16] in the case of one dimensional symmetric systems.

At the time of this writing, an open problem in the analytical theory of (1.1) is whether the formal convergence to its non-nonlocal analogue $\partial_{t} U+\operatorname{div}_{\mathbf{x}} F(t, \mathbf{x}, U, U)=$ 0 as $\eta$ tends to Dirac delta can be made rigorous. The present numerical procedures can be helpful in having some insight in this issue. To this end, we consider the classical Keyfitz-Kranzer system in 2 dimensions

$$
\left\{\begin{array}{l}
\partial_{t} U^{1}+\partial_{x}\left(U^{1} \varphi^{1}(U)\right)+\partial_{y}\left(U^{1} \varphi^{2}(U)\right)=0 \\
\partial_{t} U^{2}+\partial_{x}\left(U^{2} \varphi^{1}(U)\right)+\partial_{y}\left(U^{2} \varphi^{2}(U)\right)=0
\end{array}\right.
$$

with its non-local generalization given by

$$
\left\{\begin{array}{l}
\partial_{t} U^{1}+\partial_{x}\left(U^{1} \varphi_{1}(U * \mu)\right)+\partial_{y}\left(U^{1} \varphi_{2}(U * \mu)\right)=0 \\
\partial_{t} U^{2}+\partial_{x}\left(U^{2} \varphi_{1}(U * \mu)\right)+\partial_{y}\left(U^{2} \varphi_{2}(U * \mu)\right)=0
\end{array}\right.
$$


which is a system of 2 nonlocal conservation laws in 2 space dimensions. This system fits into the framework of Theorem 2.2.

Lemma 3.2. System (3.8) fits into (2.1) setting:

$$
\begin{array}{ll}
N=2, & f(t, x, y, U, A)=U \varphi(A) \\
m=2, & g(t, x, y, U, B)=U \varphi(B)
\end{array} \quad \text { and } \quad \eta=\vartheta=\left[\begin{array}{cc}
\mu & 0 \\
0 & \mu
\end{array}\right] .
$$

Moreover, if $\varphi_{1}, \varphi_{2} \in\left(\mathbf{C}^{2} \cap \mathbf{W}^{2, \infty}\right)\left(\mathbb{R}^{2} ; \mathbb{R}\right)$ and $\mu \in\left(\mathbf{C}^{2} \cap \mathbf{W}^{2, \infty}\right)\left(\mathbb{R}^{2} ; \mathbb{R}\right)$, then for any compactly supported initial datum $\left(U_{o}^{1}, U_{o}^{2}\right) \in\left(\mathbf{L}^{1} \cap \mathbf{L}^{\infty} \cap \mathbf{B V}\right)\left(\mathbb{R}^{2} ; \mathbb{R}_{+}^{2}\right)$, problem $(3.8)$ admits a solution defined for all $t \in \mathbb{R}^{+}$and satisfying the estimates in Theorem 2.2. The proof of this Lemma, i.e., that (H0) and (H3) hold in the case of (3.8), is immediate and, hence, omitted.

The following numerical integration of $(3.8)$ is referred to the domain $[-1.1,1.1] \times$ $[-1.1,1.1]$ and to the time interval $[0,0.1]$ with

$$
\varphi_{1}\left(A_{1}, A_{2}\right)=\sin \left({A_{1}}^{2}+{A_{2}}^{2}\right), \quad \varphi_{2}\left(B_{1}, B_{2}\right)=\cos \left(B_{1}^{2}+B_{2}^{2}\right)
$$

and

$$
\tilde{\mu}(x, y)=\left(r^{2}-\left(x^{2}+y^{2}\right)\right)^{3} \chi_{\left\{(x, y): x^{2}+y^{2} \leq r^{2}\right\}}(x, y), \quad \mu(x, y)=\frac{\tilde{\mu}(x, y)}{\iint_{\mathbb{R}^{2}} \tilde{\mu}}
$$

so that $\iint_{\mathbb{R}^{2}} \mu(x, y) \mathrm{d} x \mathrm{~d} y=1$. As initial datum, we choose:

$$
U_{o}(x, y)= \begin{cases}(1, \sqrt{3}) & (x, y) \in] 0,0.4] \times] 0,0.4] \\ (\sqrt{2}, 1) & (x, y) \in]-0.4,0] \times] 0,0.4] \\ \left(\frac{1}{2}, \frac{1}{3}\right) & (x, y) \in]-0.4,0] \times]-0.4,0] \\ (\sqrt{3}, \sqrt{2}) & (x, y) \in] 0,0.4] \times]-0.4,0] \\ (0,0) & \text { elsewhere }\end{cases}
$$

Note that the above initial datum is compactly supported. In view of Remark 1, it is then sufficient to verify (H0) for Theorem 2.2, and hence Lemma 3.2, to be applied. Corresponding to the initial datum (3.11), the solution for (3.8)-(3.10) computed
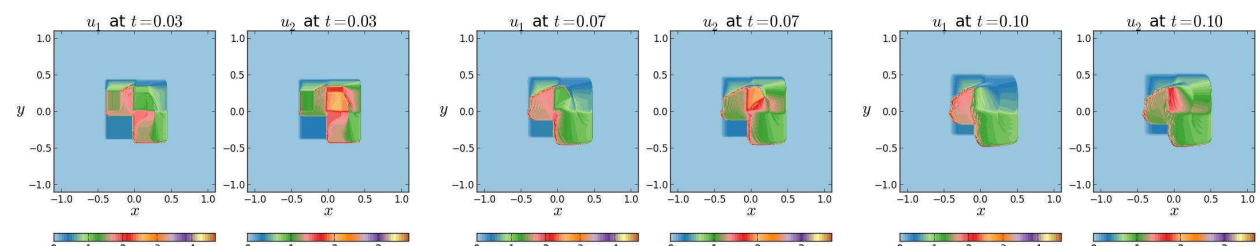

FIG. 3.2. Integration of (3.8)-(3.10) with initial datum (3.11) at times $t=0.03,0.07,0.1$.

with $r=.0125$ is depicted in Figure 3.2. The solution of the non-nonlocal analogue of (3.8)-(3.10) is shown in Figure 3.3. Both the simulations were obtained with a space mesh $\Delta x=\Delta y=1.25 E-3, \lambda_{x}=\lambda_{y}=0.1$ and time step $\Delta t=1.25 E-4$. The color scales in figures 3.2 and 3.3 range from the minimum to the maximum of the computed solutions $U^{1}$ and $U^{2}$ for (3.8)-(3.10) over the time interval [0,0.1].

We consider the following radii of the convolution kernel $\mu$, see (3.10):

$$
r=0.05,0.0375,0.025,0.0125
$$



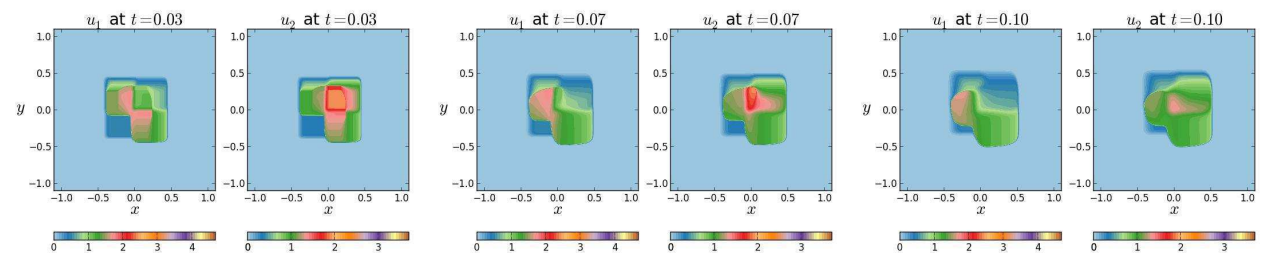

FIG. 3.3. Integration of the non-nonlocal analogue of (3.8)-(3.10) with initial datum (3.11) at times $t=0.03,0.07,0.1$.

and compute the corresponding solutions to (3.8)-(3.11). Then, we compare them with the solutions to (3.7)-(3.11). In both the integrations, $\lambda_{x}=\lambda_{y}=0.1$ and the time step is $\Delta t=1.25 E-4$. The result of this comparison is shown in Table 3.2. The present numerical integrations can not prove the convergence of the nonlocal to

\begin{tabular}{|c|c|c|c|c|c|c|c|c|}
\hline & \multicolumn{5}{|c|}{$u_{1}$} & \multicolumn{4}{|c|}{$u_{2}$} \\
\hline$r$ & 0.05 & 0.0375 & 0.025 & 0.0125 & .05 & 0.0375 & 0.025 & 0.0125 \\
\hline 0.03 & 0.2246 & 0.2236 & 0.2222 & 0.2207 & 0.2183 & 0.2177 & 0.2168 & 0.2158 \\
\hline 0.07 & 0.3589 & 0.3577 & 0.3565 & 0.3543 & 0.3476 & 0.3461 & 0.3454 & 0.3447 \\
\hline 0.10 & 0.4479 & 0.4453 & 0.4424 & 0.4384 & 0.4135 & 0.4116 & 0.4100 & 0.4077 \\
\hline
\end{tabular}

Difference of the $L^{1}$ norm of the solution $\left(u_{1}, u_{2}\right)$ to (3.7) and solutions to (3.8) with different values of $r$ as in (3.12), all with the same initial datum (3.11), with the same space meshes $\Delta x=$ $\Delta y=1.25 E-3$ and with a time step $\Delta t=1.25 E-4$.

non-nonlocal problem, nevertheless they suggest that a positive answer is possible.

4. Technical Details. Throughout, for any pair $a, b \in \mathbb{R}^{D}$, by $I(a, b)$ we denote the segment $\{a+t(b-a): t \in[0,1]\}$. We make extensive use of the following lemmas.

Lemma 4.1. Let $H, K$ and $U_{o}$ be positive. Define $u^{n+1}=(1+H) u^{n}+K$. Then,

$$
u^{n}=(1+H)^{n} U_{o}+\frac{(1+H)^{n}-1}{H} K \leq(1+H)^{n}\left(U_{o}+n K\right) .
$$

Proof. The first equality is immediate. Then, using the binomial theorem, we get

$$
\frac{(1+H)^{n}-1}{H}=\sum_{h=1}^{n}\left(\begin{array}{c}
n \\
h
\end{array}\right) H^{h-1} \leq \sum_{h=0}^{n-1} n\left(\begin{array}{c}
n-1 \\
h
\end{array}\right) H^{h} \leq n(1+H)^{n} .
$$

Lemma 4.2. Under conditions (H3), the algorithm (2.3) yields the estimates

$$
\begin{aligned}
\left\|A_{i+1 / 2, j}^{n}-A_{i-1 / 2, j}^{n}\right\| & \leq \Delta x\left\|\partial_{x} \eta\right\|_{\mathbf{L}^{\infty}}\left\|u_{\Delta}\left(t^{n}\right)\right\|_{\mathbf{L}^{1}}, \\
\left\|A_{i+1 / 2, j+1}^{n}-A_{i+1 / 2, j}^{n}\right\| & \leq \Delta y\left\|\partial_{y} \eta\right\|_{\mathbf{L}^{\infty}}\left\|u_{\Delta}\left(t^{n}\right)\right\|_{\mathbf{L}^{1}}, \\
\left\|B_{i, j+1 / 2}^{n}-B_{i, j-1 / 2}^{n}\right\| & \leq \Delta y\left\|\partial_{y} \vartheta\right\|_{\mathbf{L}^{\infty}}\left\|u_{\Delta}\left(t^{n}\right)\right\|_{\mathbf{L}^{1}}, \\
\left\|B_{i+1, j+1 / 2}^{n}-B_{i, j+1 / 2}^{n}\right\| & \leq \Delta x\left\|\partial_{x} \vartheta\right\|_{\mathbf{L}^{\infty}}\left\|u_{\Delta}\left(t^{n}\right)\right\|_{\mathbf{L}^{1}},
\end{aligned}
$$




$$
\begin{aligned}
\left\|A_{i+3 / 2, j}^{n}-2 A_{i+1 / 2, j}^{n}+A_{i-1 / 2, j}^{n}\right\| & \leq 2 \Delta x^{2}\left\|\partial_{x x}^{2} \eta\right\|_{\mathbf{L}^{\infty}}\left\|u_{\Delta}\left(t^{n}\right)\right\|_{\mathbf{L}^{1}},(4,2) \\
\left\|B_{i, j+3 / 2}^{n}-2 B_{i, j+1 / 2}^{n}+B_{i, j-1 / 2}^{n}\right\| & \leq 2 \Delta y^{2}\left\|\partial_{y y}^{2} \vartheta\right\|_{\mathbf{L}^{\infty}}\left\|u_{\Delta}\left(t^{n}\right)\right\|_{\mathbf{L}^{1}}, \\
\left\|A_{i+1 / 2, j}^{n}-A_{i+1 / 2, j+1}^{n}-A_{i-1 / 2, j}^{n}+A_{i-1 / 2, j+1}^{n}\right\| & \leq \Delta x \Delta y\left\|\partial_{x y}^{2} \eta\right\|_{\mathbf{L}^{\infty}}\left\|u_{\Delta}\left(t^{n}\right)\right\|_{\mathbf{L}^{1}}, \\
\left\|B_{i, j+1 / 2}^{n}-B_{i+1, j+1 / 2}^{n}-B_{i, j-1 / 2}^{n}+B_{i+1, j+1 / 2}^{n}\right\| & \leq \Delta x \Delta y\left\|\partial_{x y}^{2} \vartheta\right\|_{\mathbf{L}^{\infty}}\left\|u_{\Delta}\left(t^{n}\right)\right\|_{\mathbf{L}^{1}} .
\end{aligned}
$$

Proof. Consider the estimates on the $A$ terms, the others being entirely similar:

$$
\begin{aligned}
& \left\|A_{i+1 / 2, j}^{n}-A_{i-1 / 2, j}^{n}\right\| \\
\leq & \Delta x \Delta y \sum_{l, m \in \mathbb{Z}}\left\|\left(\eta_{i+1 / 2-l, j-m}-\eta_{i-1 / 2-l, j-m}\right) u_{\Delta}\left(t^{n}, x_{l+1 / 2}, y_{m}\right)\right\| \\
\leq & \Delta x \Delta y \sum_{l, m \in \mathbb{Z}}\left\|\eta_{i+1 / 2-l, j-m}-\eta_{i-1 / 2-l, j-m}\right\|\left\|u_{\Delta}\left(t^{n}, x_{l+1 / 2}, y_{m}\right)\right\| \\
\leq & \Delta x \Delta y \sum_{l, m \in \mathbb{Z}}\left\|u_{\Delta}\left(t^{n}, x_{l+1 / 2}, y_{m}\right)\right\| \int_{x_{i-1 / 2-l}}^{x_{i+1 / 2-l}}\left\|\partial_{x} \eta\left(x, y_{j-m}\right)\right\| \mathrm{d} x \\
\leq & \Delta x\left\|\partial_{x} \eta^{k}\right\|_{\mathbf{L}^{\infty}} \Delta x \Delta y \sum_{l, m \in \mathbb{Z}}\left\|u_{\Delta}\left(t^{n}, x_{l+1 / 2}, y_{m}\right)\right\| \\
\leq & \Delta x\left\|\partial_{x} \eta^{k}\right\|_{\mathbf{L}^{\infty}}\left\|u_{\Delta}\left(t^{n}\right)\right\|_{\mathbf{L}^{1}},
\end{aligned}
$$

proving the first estimate, the second one is entirely analogous. Similarly,

$$
\begin{aligned}
& \left\|A_{i+3 / 2, j}^{n}-2 A_{i+1 / 2, j}^{n}+A_{i-1 / 2, j}^{n}\right\| \\
\leq & \Delta x \Delta y \sum_{l, m \in \mathbb{Z}}\left\|u_{\Delta}\left(t^{n}, x_{l+1 / 2}, y_{m}\right)\right\|\left\|\eta_{i+3 / 2-l, j-m}-2 \eta_{i+1 / 2-l, j-m}+\eta_{i-1 / 2-l, j-m}\right\| \\
\leq & \Delta x \Delta y \sum_{l, m \in \mathbb{Z}}\left\|u_{\Delta}\left(t^{n}, x_{l+1 / 2}, y_{m}\right)\right\| \Delta x\left\|\partial_{x} \eta\left(\tau_{i+1-l}, y_{j-m}\right)-\partial_{x} \eta\left(\tau_{i-l}, y_{j-m}\right)\right\| \\
\leq & \Delta x \Delta y \sum_{l, m \in \mathbb{Z}}\left\|u_{\Delta}\left(t^{n}, x_{l+1 / 2}, y_{m}\right)\right\| \Delta x \int_{\tau_{i-l}}^{\tau_{i+1-l}}\left\|\partial_{x x}^{2} \eta\left(s, y_{j-m}\right)\right\| \mathrm{d} s \\
\leq & 2 \Delta x^{2}\left\|\partial_{x x}^{2} \eta\right\|_{\mathbf{L}^{\infty}}\left\|u_{\Delta}\left(t^{n}\right)\right\|_{\mathbf{L}^{1}}
\end{aligned}
$$

where $\left.\tau_{i-l} \in\right] x_{i-1 / 2-l}, x_{i+1 / 2-l}[$.

Proof of Lemma 2.3. In the spirit of [3, Lemma 2.5], note that (2.3) can be rewritten as follows:

$$
\begin{aligned}
u_{i j}^{k, n+1 / 2}=u_{i j}^{k, n} & -a_{i-1 / 2, j}^{k, n}\left(u_{i j}^{k, n}-u_{i-1, j}^{k, n}\right)+b_{i+1 / 2, j}^{k, n}\left(u_{i+1, j}^{k, n}-u_{i j}^{k, n}\right) \\
& -\lambda_{x}\left(F_{i+1 / 2, j}^{k, n}\left(u_{i j}^{k, n}, u_{i j}^{k, n}\right)-F_{i-1 / 2, j}^{k, n}\left(u_{i j}^{k, n}, u_{i j}^{k, n}\right)\right),
\end{aligned}
$$

where

$$
a_{i-1 / 2, j}^{k, n}=\lambda_{x} \frac{F_{i-1 / 2, j}^{k, n}\left(u_{i j}^{k, n}, u_{i j}^{k, n}\right)-F_{i-1 / 2, j}^{k, n}\left(u_{i-1, j}^{k, n}, u_{i j}^{k, n}\right)}{u_{i j}^{k, n}-u_{i-1, j}^{k, n}},
$$




$$
b_{i+1 / 2, j}^{k, n}=\lambda_{x} \frac{F_{i+1 / 2, j}^{k, n}\left(u_{i j}^{k, n}, u_{i j}^{k, n}\right)-F_{i+1 / 2, j}^{k, n}\left(u_{i j}^{k, n}, u_{i+1, j}^{k, n}\right)}{u_{i+1, j}^{k, n}-u_{i j}^{k, n}} .
$$

We now prove that $a_{i-1 / 2, j}^{k, n}$ and $b_{i+1 / 2, j}^{k, n}$ are non negative. Indeed,

$$
\begin{aligned}
a_{i-1 / 2, j}^{k, n} & =\lambda_{x} \frac{F_{i-1 / 2, j}^{k, n}\left(u_{i j}^{k, n}, u_{i j}^{k, n}\right)-F_{i-1 / 2, j}^{k, n}\left(u_{i-1, j}^{k, n}, u_{i j}^{k, n}\right)}{u_{i j}^{k, n}-u_{i-1, j}^{k, n}} \\
& =\frac{\lambda_{x}}{u_{i j}^{k, n}-u_{i-1, j}^{k, n}}\left(f_{i-1 / 2, j}^{k, n}\left(u_{i j}^{k, n}\right)-\frac{f_{i-1 / 2, j}^{k, n}\left(u_{i-1, j}^{k, n}\right)+f_{i-1 / 2, j}^{k, n}\left(u_{i j}^{k, n}\right)}{2}\right)+\frac{\alpha}{2} \\
& =\frac{\lambda_{x}}{u_{i j}^{k, n}-u_{i-1, j}^{k, n}}\left(\frac{f_{i-1 / 2, j}^{k, n}\left(u_{i j}^{k, n}\right)-f_{i-1 / 2, j}^{k, n}\left(u_{i-1, j}^{k, n}\right)}{2}\right)+\frac{\alpha}{2} \\
& =\frac{\lambda_{x} \partial_{u} f_{i-1 / 2, j}^{k, n}\left(\zeta_{i-1 / 2, j}^{k, n}\right)+\alpha}{2}
\end{aligned}
$$

for a suitable $\zeta_{i-1 / 2, j}^{k, n}$ lying between $u_{i-1, j}^{k, n}$ and $u_{i j}^{k, n}$. Hence

$$
a_{i-1 / 2, j}^{k, n} \geq \frac{-\lambda_{x}\left\|\partial_{u} f^{k}\right\|_{\mathbf{L}^{\infty}}+\alpha}{2} \geq 0, \quad a_{i-1 / 2, j}^{k, n} \leq \frac{\lambda_{x}\left\|\partial_{u} f^{k}\right\|_{\mathbf{L}^{\infty}}+\alpha}{2} \leq \frac{1}{3}
$$

by (2.6). Entirely similar computations hold for the term $b_{i-1 / 2, j}^{k, n}$, so that $1-a_{i-1 / 2, j}^{k, n}-$ $b_{i-1 / 2, j}^{k, n}, a_{i-1 / 2, j}^{k, n}$ and $b_{i-1 / 2, j}^{k, n}$ are the coefficients of a convex combination. Using (H0),

(H1) and the CFL condition (2.6) we now estimate:

$$
\begin{aligned}
& \left|F_{i+1 / 2, j}^{k, n}\left(u_{i j}^{k, n}, u_{i j}^{k, n}\right)-F_{i-1 / 2, j}^{k, n}\left(u_{i j}^{k, n}, u_{i j}^{k, n}\right)\right| \\
= & \mid f^{k}\left(t^{n}, x_{i+1 / 2}, y_{j}, u_{i j}^{k, n}, A_{i+1 / 2, j}^{n}\right)-f^{k}\left(t^{n}, x_{i+1 / 2}, y_{j}, u_{i j}^{k, n}, A_{i-1 / 2, j}^{n}\right) \\
& +f^{k}\left(t^{n}, x_{i+1 / 2}, y_{j}, u_{i j}^{k, n}, A_{i-1 / 2, j}^{n}\right)-f^{k}\left(t^{n}, x_{i-1 / 2}, y_{j}, u_{i j}^{k, n}, A_{i-1 / 2, j}^{n}\right) \mid \\
\leq & \left|f^{k}\left(t^{n}, x_{i+1 / 2}, y_{j}, u_{i j}^{k, n}, A_{i+1 / 2, j}^{n}\right)\right|+\left|f^{k}\left(t^{n}, x_{i+1 / 2}, y_{j}, u_{i j}^{k, n}, A_{i-1 / 2, j}^{n}\right)\right| \\
& \left|f^{k}\left(t^{n}, x_{i+1 / 2}, y_{j}, u_{i j}^{k, n}, A_{i-1 / 2, j}^{n}\right)-f^{k}\left(t^{n}, x_{i-1 / 2}, y_{j}, u_{i j}^{k, n}, A_{i-1 / 2, j}^{n}\right)\right| \\
\leq & 2|| \partial_{u} f^{k} \|_{\mathbf{L}_{\infty}} u_{i j}^{k, n}+\left|\partial_{x} f^{k}\left(t^{n}, \bar{x}_{i}, y_{j}, u_{i j}^{k, n}, A_{i-1 / 2, j}^{n}\right)\right| \Delta x \\
\leq & \left(2\left\|\partial_{u} f^{k}\right\|_{\mathbf{L}^{\infty}}+M \Delta x\right) u_{i j}^{k, n}
\end{aligned}
$$

for some $\left.\bar{x}_{i} \in\right] x_{i-1 / 2}, x_{i+1 / 2}[$. Now using condition (2.6) we have:

$$
\left|F_{i+1 / 2, j}^{k, n}\left(u_{i j}^{k, n}, u_{i j}^{k, n}\right)-F_{i-1 / 2, j}^{k, n}\left(u_{i j}^{k, n}, u_{i j}^{k, n}\right)\right| \leq \frac{1}{3 \lambda_{x}} u_{i j}^{k, n},
$$

Using (4.4) and (4.5) in (4.3) we get:

$$
u_{i j}^{k, n+1 / 2}=\left(1-a_{i-1 / 2, j}^{k, n}-b_{i+1 / 2, j}^{k, n}\right) u_{i j}^{k, n}+a_{i-1 / 2, j}^{k, n} u_{i-1, j}^{k, n}+b_{i+1 / 2, j}^{k, n} u_{i+1, j}^{k, n}
$$




$$
\begin{aligned}
& -\lambda_{x}\left(F_{i+1 / 2, j}^{k, n}\left(u_{i j}^{k, n}, u_{i j}^{k, n}\right)-F_{i-1 / 2, j}^{k, n}\left(u_{i j}^{k, n}, u_{i j}^{k, n}\right)\right) \\
\geq & \left(\frac{2}{3}-a_{i-1 / 2, j}^{k, n}-b_{i+1 / 2, j}^{k, n}\right) u_{i j}^{k, n}+a_{i-1 / 2, j}^{k, n} u_{i-1, j}^{k, n}+b_{i+1 / 2, j}^{k, n} u_{i+1, j}^{k, n} \geq 0,
\end{aligned}
$$

completing the proof.

Proof of Lemma 2.5. Refer to (4.3). Using (4.1) and (H1) in (4.3), we obtain:

$$
\begin{aligned}
& F_{i+1 / 2, j}^{k, n}\left(u_{i j}^{k, n}, u_{i j}^{k, n}\right)-F_{i-1 / 2, j}^{k, n}\left(u_{i j}^{k, n}, u_{i j}^{k, n}\right) \\
= & f^{k}\left(t^{n}, x_{i+1 / 2}, y_{j}, u_{i j}^{k, n}, A_{i+1 / 2, j}^{n}\right)-f^{k}\left(t^{n}, x_{i-1 / 2}, y_{j}, u_{i j}^{k, n}, A_{i-1 / 2, j}^{n}\right) \\
= & \partial_{x} f^{k}\left(t^{n}, \bar{x}_{i}, y_{j}, u_{i j}^{k, n}, \bar{A}_{i j}^{n}\right) \Delta x+\nabla_{A} f^{k}\left(t^{n}, \bar{x}_{i}, y_{j}, u_{i j}^{k, n}, \bar{A}_{i j}^{n}\right)\left(A_{i+1 / 2, j}^{n}-A_{i-1 / 2, j}^{n}\right) \\
\leq & \left\|\nabla_{A} f^{k}\left(t^{n}, \bar{x}_{i}, y_{j}, u_{i j}^{k, n}, \bar{A}_{i j}^{n}\right)\right\|\left\|A_{i+1 / 2, j}^{n}-A_{i-1 / 2, j}^{n}\right\|+M\left|u_{i j}^{k, n}\right| \Delta x \\
\leq & M\left|u_{i j}^{k, n}\right| \Delta x\left(\left\|\partial_{x} \eta\right\|_{\mathbf{L}^{\infty}}\left\|u_{\Delta}\left(t^{n}\right)\right\|_{\mathbf{L}^{1}}+1\right)
\end{aligned}
$$

Now using Lemma 2.4, (4.4) and (4.6) in (4.3), we get

$$
\begin{aligned}
&\left|u_{i j}^{k, n+1 / 2}\right| \leq\left(1-a_{i-1 / 2, j}^{k, n}-b_{i, j}^{k, n}\right)\left|u_{i j}^{k, n}\right|+a_{i-1 / 2, j}^{k, n}\left|u_{i-1, j}^{k, n}\right| \\
& \quad+b_{i+1 / 2, j}^{k, n}\left|u_{i+1, j}^{k, n}\right|+M\left|u_{i j}^{k, n}\right| \Delta t\left(\left\|\partial_{x} \eta\right\|_{\mathbf{L}^{\infty}}\left\|u_{\Delta}\left(t^{n}\right)\right\|_{\mathbf{L}^{1}}+1\right) \\
& \leq\left(1-a_{i-1 / 2, j}^{k, n}-b_{i+1 / 2, j}^{k, n}\right)\left\|u_{\Delta}\left(t^{n}\right)\right\|_{\mathbf{L}^{\infty}}+a_{i-1 / 2, j}^{k, n}\left\|u_{\Delta}\left(t^{n}\right)\right\|_{\mathbf{L}^{\infty}} \\
& \quad+b_{i+1 / 2, j}^{k, n}\left\|u_{\Delta}\left(t^{n}\right)\right\|_{\mathbf{L}^{\infty}}+M\left\|u_{\Delta}\left(t^{n}\right)\right\|_{\mathbf{L}^{\infty}} \Delta t\left(\left\|\partial_{x} \eta\right\|_{\mathbf{L}^{\infty}}\left\|U_{o}\right\|_{\mathbf{L}^{1}}+1\right) \\
& \leq\left\|u_{\Delta}\left(t^{n}\right)\right\|_{\mathbf{L}^{\infty}}\left(1+M \Delta t\left(\left\|\partial_{x} \eta\right\|_{\mathbf{L}^{\infty}}\left\|U_{o}\right\|_{\mathbf{L}^{1}}+1\right)\right)
\end{aligned}
$$

which implies that

$$
\left\|u_{\Delta}\left(t^{n+1 / 2}\right)\right\|_{\mathbf{L}^{\infty}} \leq\left(1+M\left(\left\|\partial_{x} \eta\right\|_{\mathbf{L}^{\infty}}\left\|U_{o}\right\|_{\mathbf{L}^{1}}+1\right) \Delta t\right)\left\|u_{\Delta}\left(t^{n}\right)\right\|_{\mathbf{L}^{\infty}} .
$$

Applying the same procedure for the $y$ component gives

$$
\left\|u_{\Delta}\left(t^{n+1}\right)\right\|_{\mathbf{L}^{\infty}} \leq\left(1+M\left(\left\|\partial_{y} \vartheta\right\|_{\mathbf{L}^{\infty}}\left\|U_{o}\right\|_{\mathbf{L}^{1}}+1\right) \Delta t\right)\left\|u_{\Delta}\left(t^{n+1 / 2}\right)\right\|_{\mathbf{L}^{\infty}},
$$

and by Lemma 4.1 we have the $\mathbf{L}^{\infty}$ bound

$$
\left\|u_{\Delta}\left(t^{n}\right)\right\|_{\mathbf{L}^{\infty}} \leq\left\|U_{o}\right\|_{\mathbf{L}^{\infty}}\left[1+M\left(\max \left\{\left\|\partial_{x} \eta\right\|_{\mathbf{L}^{\infty}},\left\|\partial_{y} \vartheta\right\|_{\mathbf{L}^{\infty}}\right\}\left\|U_{o}\right\|_{\mathbf{L}^{1}}+1\right) \Delta t\right]^{2 n},
$$

so that (2.7) holds with

$$
\mathcal{C}=2 M\left(1+\max \left\{\left\|\partial_{x} \eta\right\|_{\mathbf{L}^{\infty}},\left\|\partial_{y} \vartheta\right\|_{\mathbf{L}^{\infty}}\right\}\right)
$$

completing the proof. 
Proof of Lemma 2.6. We follow the proof of [3, Proposition 2.6]. Consider first the term $\sum_{i j}\left|u_{i+1, j}^{k, n+1 / 2}-u_{i j}^{k, n+1 / 2}\right| \Delta y$, setting $u_{i+1, j}^{k, n+1 / 2}-u_{i j}^{k, n+1 / 2}=C_{i j}^{n}-\lambda_{x} D_{i j}^{n}$, where

$$
\begin{gathered}
C_{i j}^{n}=\left(u_{i+1, j}^{k, n}-u_{i j}^{k, n}\right)-\lambda_{x}\left(F_{i+3 / 2, j}^{k, n}\left(u_{i+1, j}^{k, n}, u_{i+2, j}^{k, n}\right)-F_{i+1 / 2, j}^{k, n}\left(u_{i j}^{k, n}, u_{i+1, j}^{k, n}\right)\right) \\
\quad \lambda_{x}\left(-F_{i+3 / 2, j}^{k, n}\left(u_{i j}^{k, n}, u_{i+1, j}^{k, n}\right)+F_{i+1 / 2, j}^{k, n}\left(u_{i-1, j}^{k, n}, u_{i j}^{k, n}\right)\right) \\
D_{i j}^{n}=F_{i+3 / 2, j}^{k, n}\left(u_{i j}^{k, n}, u_{i+1, j}^{k, n}\right)-F_{i+1 / 2, j}^{k, n}\left(u_{i j}^{k, n}, u_{i+1, j}^{k, n}\right) \\
\quad+F_{i-1 / 2, j}^{k, n}\left(u_{i-1, j}^{k, n}, u_{i j}^{k, n}\right)-F_{i+1 / 2, j}^{k, n}\left(u_{i-1, j}^{k, n}, u_{i j}^{k, n}\right)
\end{gathered}
$$

and we now estimate the various terms above separately. Consider the term $C_{i j}^{n}$.

$$
\begin{aligned}
C_{i j}^{n}= & \left(u_{i+1, j}^{k, n}-u_{i j}^{k, n}\right)\left(1+\lambda_{x} \frac{F_{i+1 / 2, j}^{k, n}\left(u_{i j}^{k, n}, u_{i+1, j}^{k, n}\right)-F_{i+1 / 2, j}^{k, n}\left(u_{i j}^{k, n}, u_{i j}^{k, n}\right)}{u_{i+1, j}^{k, n}-u_{i j}^{k, n}}\right) \\
& +\left(u_{i+1, j}^{k, n}-u_{i j}^{k, n}\right)\left(-\lambda_{x} \frac{F_{i+3 / 2, j}^{k, n}\left(u_{i+1, j}^{k, n}, u_{i+1, j}^{k, n}\right)-F_{i+3 / 2, j}^{k, n}\left(u_{i j}^{k, n}, u_{i+1, j}^{k, n}\right)}{u_{i+1, j}^{k, n}-u_{i j}^{k, n}}\right) \\
& +\left(u_{i+2, j}^{k, n}-u_{i+1, j}^{k, n}\right)\left(-\lambda_{x} \frac{F_{i+3 / 2, j}^{k, n}\left(u_{i+1, j}^{k, n}, u_{i+2, j}^{k, n}\right)-F_{i+3 / 2, j}^{k, n}\left(u_{i+1, j}^{k, n}, u_{i+1, j}^{k, n}\right)}{u_{i+2, j}^{k, n}-u_{i+1, j}^{k, n}}\right) \\
& +\left(u_{i j}^{k, n}-u_{i-1, j}^{k, n}\right)\left(\lambda_{x} \frac{F_{i+1 / 2, j}^{k, n}\left(u_{i j}^{k, n}, u_{i j}^{k, n}\right)-F_{i+1 / 2, j}^{k, n}\left(u_{i-1, j}^{k, n}, u_{i j}^{k, n}\right)}{u_{i j}^{k, n}-u_{i-1, j}^{k, n}}\right) \\
= & \left(u_{i+1, j}^{k, n}-u_{i j}^{k, n}\right)\left(1-b_{i+\frac{1}{2}, j}^{k, n}-\tilde{a}_{i+\frac{1}{2}, j}^{k, n}\right)+\left(u_{i+2, j}^{k, n}-u_{i+1, j}^{k, n}\right) b_{i+\frac{3}{2}, j}^{k, n} \\
& +\left(u_{i j}^{k, n}-u_{i-1, j}^{k, n}\right) \tilde{a}_{i-\frac{1}{2}, j}^{k, n}
\end{aligned}
$$

where $\tilde{a}_{i-\frac{1}{2}, j}^{k, n}=\lambda_{x}\left[F_{i+1 / 2, j}^{k, n}\left(u_{i j}^{k, n}, u_{i j}^{k, n}\right)-F_{i+1 / 2, j}^{k, n}\left(u_{i-1, j}^{k, n}, u_{i j}^{k, n}\right)\right] /\left(u_{i j}^{k, n}-u_{i-1, j}^{k, n}\right)$ and $\tilde{a}_{i-\frac{1}{2}, j}^{k, n} \in[0,1 / 3]$ can be proved exactly as was done for $a_{i-\frac{1}{2}, j}^{k, n}$. By convexity, we have

$$
\sum_{i j}\left|C_{i j}^{n}\right| \leq \sum_{i j}\left|u_{i+1, j}^{k, n}-u_{i j}^{k, n}\right|
$$

Concerning $D_{i j}^{n}$,

$$
\begin{aligned}
D_{i j}^{n}= & \frac{1}{2}\left(f_{i+3 / 2, j}^{k, n}\left(u_{i j}^{k, n}\right)-2 f_{i+1 / 2, j}^{k, n}\left(u_{i j}^{k, n}\right)+f_{i-1 / 2, j}^{k, n}\left(u_{i j}^{k, n}\right)\right) \\
& +\frac{1}{2}\left(f_{i+3 / 2, j}^{k, n}\left(u_{i+1, j}^{k, n}\right)-f_{i+1 / 2, j}^{k, n}\left(u_{i+1, j}^{k, n}\right)\right) \\
& -\frac{1}{2}\left(f_{i+1 / 2, j}^{k, n}\left(u_{i-1, j}^{k, n}\right)-f_{i-1 / 2, j}^{k, n}\left(u_{i-1, j}^{k, n}\right)\right) .
\end{aligned}
$$

Let us first consider the estimates (4.10) and (4.11):

$$
\begin{aligned}
& (4.10)+(4.11) \\
= & \frac{1}{2}\left(f^{k}\left(t^{n}, x_{i+3 / 2}, y_{j}, u_{i+1, j}^{k, n}, A_{i+3 / 2, j}^{n}\right)-f^{k}\left(t^{n}, x_{i+1 / 2}, y_{j}, u_{i+1, j}^{k, n}, A_{i+3 / 2, j}^{n}\right)\right)
\end{aligned}
$$




$$
\begin{aligned}
& +\frac{1}{2}\left(f^{k}\left(t^{n}, x_{i+1 / 2}, y_{j}, u_{i+1, j}^{k, n}, A_{i+3 / 2, j}^{n}\right)-f^{k}\left(t^{n}, x_{i+1 / 2}, y_{j}, u_{i+1, j}^{k, n}, A_{i+1 / 2, j}^{n}\right)\right) \\
& -\frac{1}{2}\left(f^{k}\left(t^{n}, x_{i+1 / 2}, y_{j}, u_{i-1, j}^{k, n}, A_{i+1 / 2, j}^{n}\right)-f^{k}\left(t^{n}, x_{i-1 / 2}, y_{j}, u_{i-1, j}^{k, n}, A_{i+1 / 2, j}^{n}\right)\right) \\
& -\frac{1}{2}\left(f^{k}\left(t^{n}, x_{i-1 / 2}, y_{j}, u_{i-1, j}^{k, n}, A_{i+1 / 2, j}^{n}\right)-f^{k}\left(t^{n}, x_{i-1 / 2}, y_{j}, u_{i-1, j}^{k, n}, A_{i-1 / 2, j}^{n}\right)\right) \\
= & \frac{1}{2} \Delta x\left(\partial_{x} f^{k}\left(t^{n}, \bar{x}_{i+1}, y_{j}, u_{i+1, j}^{k, n}, A_{i+3 / 2, j}^{n}\right)-\partial_{x} f^{k}\left(t^{n}, \bar{x}_{i}, y_{j}, u_{i-1, j}^{k, n}, A_{i+1 / 2, j}^{n}\right)\right) \\
& +\frac{1}{2}\left(\nabla_{A} f^{k}\left(t^{n}, x_{i+1 / 2}, y_{j}, u_{i+1, j}^{k, n}, \bar{A}_{i+1, j}^{n}\right)\left(A_{i+3 / 2, j}^{n}-A_{i+1 / 2, j}^{n}\right)\right) \\
& -\frac{1}{2}\left(\nabla_{A} f^{k}\left(t^{n}, x_{i-1 / 2}, y_{j}, u_{i-1, j}^{k, n}, \bar{A}_{i, j}^{n}\right)\left(A_{i+1 / 2, j}^{n}-A_{i-1 / 2, j}^{n}\right)\right)
\end{aligned}
$$

for suitable $\left.\bar{x}_{i} \in\right] x_{i-1 / 2}, x_{i+1 / 2}\left[\right.$ and $\bar{A}_{i, j}^{n} \in I\left(A_{i-1 / 2, j}^{n}, A_{i+1 / 2, j}^{n}\right)$. Introduce $\hat{x}_{i+1 / 2} \in$ ] $\bar{x}_{i}, \bar{x}_{i+1}\left[, \check{x}_{i} \in\right] x_{i-1 / 2}, x_{i+1 / 2}\left[, \bar{u}_{i j}^{k, n} \in I\left(u_{i-1, j}^{k, n}, u_{i+1, j}^{k, n}\right), \check{u}_{i j}^{k, n} \in I\left(u_{i-1, j}^{k, n}, u_{i+1, j}^{k, n}\right)\right.$, $\hat{A}_{i, j}^{n} \in I\left(A_{i-1 / 2, j}^{n}, A_{i+1 / 2, j}^{n}\right), \check{A}_{i+1 / 2, j}^{n} \in I\left(\bar{A}_{i, j}^{n}, \bar{A}_{i+1, j}^{n}\right)$ and $\tilde{A}_{i+1 / 2, j}^{n} \in I\left(A_{i+1 / 2, j}^{n}-\right.$ $\left.A_{i-1 / 2, j}^{n}, A_{i+3 / 2, j}^{n}-A_{i+1 / 2, j}^{n}\right)$ so that we have

$$
\begin{aligned}
\mid(4.10) & +(4.11) \mid \\
\leq \frac{\Delta x}{2} & \left(\Delta x\left|\partial_{x x}^{2} f^{k}\left(t^{n}, \hat{x}_{i+1 / 2}, y_{j}, \bar{u}_{i, j}^{k, n}, \hat{A}_{i+1, j}^{n}\right)\right|\right. \\
& +\left|\partial_{x} \partial_{u} f^{k}\left(t^{n}, \hat{x}_{i+1 / 2}, y_{j}, \bar{u}_{i, j}^{k, n}, \hat{A}_{i+1, j}^{n}\right)\right|\left|u_{i+1, j}^{k, n}-u_{i-1, j}^{k, n}\right| \\
& \left.+\left\|\partial_{x} \nabla_{A} f^{k}\left(t^{n}, \hat{x}_{i+1 / 2}, y_{j}, \bar{u}_{i, j}^{k, n}, \hat{A}_{i+1, j}^{n}\right)\right\|\left\|A_{i+3 / 2, j}^{n}-A_{i+1 / 2, j}^{n}\right\|\right) \\
+\frac{1}{2}( & \Delta x\left\|\partial_{x} \nabla_{A} f^{k}\left(t^{n}, \check{x}_{i}, y_{j}, \check{u}_{i, j}^{k, n}, \check{A}_{i+1 / 2, j}^{n}\right)\right\|\left\|\tilde{A}_{i+\frac{1}{2}, j}^{n}\right\| \\
& +\left\|\partial_{u} \nabla_{A} f^{k}\left(t^{n}, \check{x}_{i}, y_{j}, \check{u}_{i, j}^{k, n}, \check{A}_{i+1 / 2, j}^{n}\right)\right\|\left\|\tilde{A}_{i+\frac{1}{2}, j}^{n}\right\|\left|u_{i+1, j}^{k, n}-u_{i-1, j}^{k, n}\right| \\
& +\left\|\nabla_{A A}^{2} f^{k}\left(t^{n}, \check{x}_{i}, y_{j}, \check{u}_{i, j}^{k, n}, \check{A}_{i+1 / 2, j}^{n}\right)\right\|\left\|\tilde{A}_{i+\frac{1}{2}, j}^{n}\right\|\left\|\bar{A}_{i+1, j}^{n}-\bar{A}_{i, j}^{n}\right\| \\
& \left.+\left\|\nabla_{A} f^{k}\left(t^{n}, \check{x}_{i}, y_{j}, \check{u}_{i, j}^{k, n}, \check{A}_{i+1 / 2, j}^{n}\right)\right\|\left\|A_{i+3 / 2, j}^{n}-2 A_{i+3 / 2, j}^{n}+A_{i-1 / 2, j}^{n}\right\|\right)
\end{aligned}
$$

By $\tilde{A}_{i+\frac{1}{2}, j}^{n} \in I\left(A_{i+1 / 2, j}^{n}-A_{i-1 / 2, j}^{n}, A_{i+3 / 2, j}^{n}-A_{i+1 / 2, j}^{n}\right)$ and $\bar{A}_{i, j}^{n} \in I\left(A_{i-1 / 2, j}^{n}, A_{i+1 / 2, j}^{n}\right)$, using Lemma 4.2, we have $\left\|\tilde{A}_{i+\frac{1}{2}, j}^{n}\right\| \leq \Delta x\left\|\partial_{x} \eta\right\|_{\mathbf{L}^{\infty}}\left\|u_{\Delta}\left(t^{n}\right)\right\|_{\mathbf{L}^{1}}$ and $\left\|\bar{A}_{i+1, j}^{n}-\bar{A}_{i, j}^{n}\right\| \leq \Delta x\left\|\partial_{x} \eta\right\|_{\mathbf{L}^{\infty}}\left\|u_{\Delta}\left(t^{n}\right)\right\|_{\mathbf{L}^{1}}$.

Now using Lemma 2.4, we have

$$
\begin{aligned}
& \quad|(4.10)+(4.11)| \\
& \leq \frac{1}{2}\left[M \Delta x^{2}\left|\bar{u}_{i, j}^{k, n}\right|+\Delta x\left\|\partial_{x} \partial_{u} f\right\|_{\mathbf{L}^{\infty}}\left|u_{i+1, j}^{k, n}-u_{i-1, j}^{k, n}\right|+M \Delta x^{2}\left|\bar{u}_{i, j}^{k, n}\right|\left\|\partial_{x} \eta\right\|_{\mathbf{L}^{\infty}}\left\|U_{o}\right\|_{\mathbf{L}^{1}}\right] \\
& +\frac{1}{2}\left[M \Delta x^{2}\left|\check{u}_{i, j}^{k, n}\right|\left\|\partial_{x} \eta\right\|_{\mathbf{L}^{\infty}}\left\|U_{o}\right\|_{\mathbf{L}^{1}}+\Delta x\left\|\partial_{u} \nabla_{A} f\right\|_{\mathbf{L}^{\infty}}\left\|\partial_{x} \eta\right\|_{\mathbf{L}^{\infty}}\left\|U_{o}\right\|_{\mathbf{L}^{1}}\left|u_{i+1, j}^{k, n}-u_{i-1, j}^{k, n}\right|\right. \\
& \left.\quad+M \Delta x^{2}\left|\check{u}_{i, j}^{k, n}\right|\left\|\partial_{x} \eta\right\|_{\mathbf{L}^{\infty}}^{2}\left\|U_{o}\right\|_{\mathbf{L}^{1}}^{2}+2 M \Delta x^{2}\left|\check{u}_{i, j}^{k, n}\right|\left\|\partial_{x x}^{2} \eta\right\|_{\mathbf{L}^{\infty}}\left\|U_{o}\right\|_{\mathbf{L}^{1}}\right]
\end{aligned}
$$




$$
\begin{aligned}
= & \frac{1}{2} M \Delta x^{2}\left|\bar{u}_{i, j}^{k, n}\right|\left(1+\left\|\partial_{x} \eta\right\|_{\mathbf{L}^{\infty}}\left\|U_{o}\right\|_{\mathbf{L}^{1}}\right) \\
& +\frac{1}{2} M \Delta x^{2}\left|\check{u}_{i, j}^{k, n}\right|\left(\left\|\partial_{x} \eta\right\|_{\mathbf{L}^{\infty}}^{2}\left\|U_{o}\right\|_{\mathbf{L}^{1}}^{2}+2\left\|\partial_{x x}^{2} \eta\right\|_{\mathbf{L}^{\infty}}\left\|U_{o}\right\|_{\mathbf{L}^{1}}+\left\|\partial_{x} \eta\right\|_{\mathbf{L}^{\infty}}\left\|U_{o}\right\|_{\mathbf{L}^{1}}\right) \\
& +\frac{1}{2} \Delta x\left|u_{i+1, j}^{k, n}-u_{i-1, j}^{k, n}\right|\left(\left\|\partial_{u} \nabla_{A} f\right\|_{\mathbf{L}^{\infty}}\left\|\partial_{x} \eta\right\|_{\mathbf{L}^{\infty}}\left\|U_{o}\right\|_{\mathbf{L}^{1}}+\left\|\partial_{x} \partial_{u} f\right\|_{\mathbf{L}^{\infty}}\right) .
\end{aligned}
$$

Now, let us estimate (4.9).

$$
\begin{aligned}
& \frac{1}{2}\left(f_{i+3 / 2, j}^{k, n}\left(u_{i j}^{k, n}\right)-2 f_{i+1 / 2, j}^{k, n}\left(u_{i j}^{k, n}\right)+f_{i-1 / 2, j}^{k, n}\left(u_{i j}^{k, n}\right)\right) \\
= & \frac{1}{2}\left(f^{k}\left(t^{n}, x_{i+3 / 2}, y_{j}, u_{i j}^{k, n}, a_{i+3 / 2, j}^{k, n}\right)-2 f^{k}\left(t^{n}, x_{i+1 / 2}, y_{j}, u_{i j}^{k, n}, a_{i+1 / 2, j}^{k, n}\right)\right. \\
& \left.+f^{k}\left(t^{n}, x_{i-1 / 2}, y_{j}, u_{i j}^{k, n}, a_{i-1 / 2, j}^{k, n}\right)\right) \\
= & \frac{1}{2} \Delta x\left(\partial_{x} f^{k}\left(t^{n}, \breve{x}_{i+1}, y_{j}, u_{i j}^{k, n}, \breve{A}_{i+1, j}^{n}\right)-\partial_{x} f^{k}\left(t^{n}, \breve{x}_{i}, y_{j}, u_{i j}^{k, n}, \breve{A}_{i j}^{n}\right)\right) \\
& +\frac{1}{2} \nabla_{A} f^{k}\left(t^{n}, \breve{x}_{i+1}, y_{j}, u_{i j}^{k, n}, \breve{A}_{i+1, j}^{n}\right)\left(A_{i+3 / 2, j}^{n}-A_{i+1 / 2}^{n}\right) \\
& -\frac{1}{2} \nabla_{A} f^{k}\left(t^{n}, \breve{x}_{i}, y_{j}, u_{i j}^{k, n}, \breve{A}_{i j}^{n}\right)\left(A_{i+1 / 2, j}^{n}-A_{i-1 / 2}^{n}\right) \\
= & \frac{1}{2} \Delta x\left[\Delta x \partial_{x x}^{2} f^{k}\left(t^{n}, \dot{x}_{i+1 / 2}, y_{j}, u_{i j}^{k, n}, \grave{A}_{i+1 / 2, j}^{n}\right)\right. \\
& \left.\quad+\partial_{x} \nabla_{A} f^{k}\left(t^{n}, \dot{x}_{i+1 / 2}, y_{j}, u_{i j}^{k, n}, \hat{A}_{i+1 / 2, j}^{n}\right)\left(\breve{A}_{i+1, j}^{n}-\breve{A}_{i j}^{n}\right)\right] \\
& +\frac{1}{2} \Delta x \partial_{x} \nabla_{A} f^{k}\left(t^{n}, \grave{x}_{i+1 / 2}, y_{j}, u_{i j}^{k, n}, \grave{A}_{i+1 / 2, j}^{n}\right) \tilde{A}_{i+1 / 2, j}^{n} \\
& +\frac{1}{2} \nabla_{A A}^{2} f^{k}\left(t^{n}, \grave{x}_{i+1 / 2}, y_{j}, u_{i j}^{k, n}, \grave{A}_{i+1 / 2, j}^{n}\right) \tilde{A}_{i+1 / 2, j}^{n}\left(\breve{A}_{i+1, j}^{n}-\breve{A}_{i j}^{n}\right) \\
& +\frac{1}{2} \nabla_{A} f^{k}\left(t^{n}, \grave{x}_{i+1 / 2}, y_{j}, u_{i j}^{k, n}, \grave{A}_{i+1 / 2, j}^{n}\right)\left(A_{i+3 / 2, j}^{n}-2 A_{i+1 / 2, j}^{n}+A_{i-1 / 2, j}^{n}\right),
\end{aligned}
$$

for suitable $\left.\breve{x}_{i} \in\right] x_{i-1 / 2}, x_{i+1 / 2}\left[, \breve{A}_{i, j}^{n} \in I\left(A_{i-1 / 2, j}^{n}, A_{i+1 / 2, j}^{n}\right), \dot{x}_{i}, \grave{x}_{i} \in\right] \breve{x}_{i}, \breve{x}_{i+1}[$, $\hat{A}_{i, j}^{n}, \grave{A}_{i, j}^{n} \in I\left(\breve{A}_{i, j}^{n}, \breve{A}_{i+1, j}^{n}\right)$, and $\widetilde{A}_{i+1 / 2, j}^{n} \in I\left(A_{i+1 / 2, j}^{n}-A_{i-1 / 2}^{n}, A_{i+3 / 2, j}^{n}-A_{i+1 / 2}^{n}\right)$. Passing to the modulus,

$$
\begin{aligned}
|(4.9)| \leq & \frac{1}{2} \Delta x^{2} M\left|u_{i j}^{k, n}\right|+\Delta x^{2} M\left|u_{i j}^{k, n}\right|\left\|\partial_{x} \eta\right\|_{\mathbf{L}^{\infty}}\left\|U_{o}\right\|_{\mathbf{L}^{1}} \\
& +\frac{1}{2} \Delta x^{2} M\left|u_{i j}^{k, n}\right|\left(\left\|\partial_{x} \eta\right\|_{\mathbf{L}^{\infty}}\left\|U_{o}\right\|_{\mathbf{L}^{1}}\right)^{2}+\Delta x^{2} M\left|u_{i j}^{k, n}\right|\left\|\partial_{x x}^{2} \eta\right\|_{\mathbf{L}^{\infty}}\left\|U_{o}\right\|_{\mathbf{L}^{1}}
\end{aligned}
$$

so that

$$
\begin{aligned}
\sum_{i j} \lambda_{x}\left|D_{i j}^{n}\right| \Delta y & \leq \sum_{i j} \lambda_{x}(|(4.9)|+|(4.10)+(4.11)|) \Delta y \\
& \leq \Delta t\left(K+K_{1} \sum_{i j}\left|u_{i+1, j}^{k, n}-u_{i j}^{k, n}\right| \Delta y\right)
\end{aligned}
$$

and, adding up (4.8) to the latter expression above

$$
\sum_{i j}\left|u_{i+1, j}^{k, n+1 / 2}-u_{i j}^{k, n+1 / 2}\right| \Delta y \leq\left(1+K_{1} \Delta t\right) \sum_{i j}\left|u_{i+1, j}^{k, n}-u_{i j}^{k, n}\right| \Delta y+K \Delta t .
$$


where

$$
\begin{aligned}
K & =M\left\|U_{o}\right\|_{\mathbf{L}^{1}}\left[1+2\left\|U_{o}\right\|_{\mathbf{L}^{1}}\left(\left\|\partial_{x} \eta\right\|_{\mathbf{L}^{\infty}}+\left\|\partial_{x x}^{2} \eta\right\|_{\mathbf{L}^{\infty}}\right)+\left\|\partial_{x} \eta\right\|_{\mathbf{L}^{\infty}}^{2}\left\|U_{o}\right\|_{\mathbf{L}^{1}}^{2}\right] \\
K_{1} & =\left\|\partial_{u} \nabla_{A} f\right\|_{\mathbf{L}^{\infty}}\left\|\partial_{x} \eta\right\|_{\mathbf{L}^{\infty}}\left\|U_{o}\right\|_{\mathbf{L}^{1}}+\left\|\partial_{x} \partial_{u} f\right\|_{\mathbf{L}^{\infty}}
\end{aligned}
$$

We now deal with $\sum_{i j}\left|u_{i, j+1}^{k, n+1 / 2}-u_{i j}^{k, n+1 / 2}\right| \Delta x$, setting $u_{i, j+1}^{k, n+1 / 2}-u_{i j}^{k, n+1 / 2}=\tilde{C}_{i j}^{n}+$ $\lambda_{x} \tilde{D}_{i j}^{n}$, where

$$
\begin{aligned}
\tilde{C}_{i j}^{n}= & \left(u_{i, j+1}^{k, n}-u_{i j}^{k, n}\right)-\lambda_{x}\left(F_{i+1 / 2, j}^{k, n}\left(u_{i, j+1}^{k, n}, u_{i+1, j+1}^{k, n}\right)-F_{i+1 / 2, j}^{k, n}\left(u_{i, j}^{k, n}, u_{i+1, j}^{k, n}\right)\right) \\
& \quad-\lambda_{x}\left(F_{i-1 / 2, j+1}^{k, n}\left(u_{i-1, j}^{k, n}, u_{i, j}^{k, n}\right)-F_{i-1 / 2, j+1}^{k, n}\left(u_{i-1, j+1}^{k, n}, u_{i, j+1}^{k, n}\right)\right) \\
\tilde{D}_{i j}^{n}= & F_{i+1 / 2, j}^{k, n}\left(u_{i, j+1}^{k, n}, u_{i+1, j+1}^{k, n}\right)-F_{i+1 / 2, j+1}^{k, n}\left(u_{i, j+1}^{k, n}, u_{i+1, j+1}^{k, n}\right) \\
+ & F_{i-1 / 2, j+1}^{k, n}\left(u_{i-1, j}^{k, n}, u_{i j}^{k, n}\right)-F_{i-1 / 2, j}^{k, n}\left(u_{i-1, j}^{k, n}, u_{i j}^{k, n}\right) .
\end{aligned}
$$

Following the above treatment of $C_{i j}^{n}$, we obtain:

$$
\begin{aligned}
\tilde{C}_{i j}^{n} & =\left(u_{i, j+1}^{k, n}-u_{i j}^{k, n}\right)\left(1-\lambda_{x} \frac{F_{i+1 / 2, j}^{k, n}\left(u_{i j}^{k, n}, u_{i+1, j}^{k, n}\right)-F_{i+1 / 2, j}^{k, n}\left(u_{i, j+1}^{k, n}, u_{i+1, j}^{k, n}\right)}{u_{i, j+1}^{n}-u_{i j}^{n}}\right) \\
& +\left(u_{i, j+1}^{k, n}-u_{i j}^{k, n}\right)\left(\lambda_{x} \frac{F_{i-1 / 2, j+1}^{k, n}\left(u_{i-1, j}^{k, n}, u_{i+1, j+1}^{k, n}\right)-F_{i-1 / 2, j+1}^{k, n}\left(u_{i-1, j}^{k, n}, u_{i j}^{k, n}\right)}{u_{i, j+1}^{k, n}-u_{i j}^{k, n}}\right) \\
& +\left(u_{i-1, j+1}^{k, n}-u_{i-1, j}^{k, n}\right)\left[\lambda_{x} \frac{F_{i-1 / 2, j+1}^{k, n}\left(u_{i-1, j+1}^{k, n}, u_{i, n+1}^{k, n}\right)-F_{i-1 / 2, j+1}^{k, n}\left(u_{i-1, j}^{k, n}, u_{i, j+1}^{k, n}\right)}{u_{i-1, j+1}^{k, n}-u_{i-1, j}^{k, n}}\right] \\
& +\left(u_{i+1, j+1}^{k, n}-u_{i+1, j}^{k, n}\right)\left(-\lambda_{x} \frac{F_{i+1 / 2, j}^{k, n}\left(u_{i, j+1}^{k, n}, u_{i+1, j}^{k, n}\right)-F_{i+1 / 2, j}^{k, n}\left(u_{i, j+1}^{k, n}, u_{i+1, j+1}^{k, n}\right)}{u_{i+1, j+1}^{k, n}-u_{i+1, j}^{k, n}}\right) \\
& =\left(u_{i, j+1}^{k, n}-u_{i j}^{k, n}\right)\left(1-\bar{a}_{i j}^{k, n}-\bar{b}_{i j}^{k, n}\right)+\left(u_{i-1, j+1}^{k, n}-u_{i-1, j}^{k, n} \bar{b}_{i j}^{k, n}+\left(u_{i+1, j+1}^{k, n}-u_{i+1, j}^{k, n}\right) \bar{a}_{i j}^{k, n}\right.
\end{aligned}
$$

where

$$
\begin{aligned}
& \bar{a}_{i j}^{k, n}=\lambda_{x} \frac{F_{i+1 / 2, j}^{k, n}\left(u_{i j}^{k, n}, u_{i+1, j}^{k, n}\right)-F_{i+1 / 2, j}^{k, n}\left(u_{i . j+1}^{k, n}, u_{i+1, j}^{k, n}\right)}{u_{i, j+1}^{n}-u_{i j}^{n}} \\
& \bar{b}_{i j}^{k, n}=-\lambda_{x} \frac{F_{i-1 / 2, j+1}^{k, n}\left(u_{i-1, j}^{k, n}, u_{i+1, j+1}^{k, n}\right)-F_{i-1 / 2, j+1}^{k, n}\left(u_{i-1, j}^{k, n}, u_{i j}^{k, n}\right)}{u_{i, j+1}^{k, n}-u_{i j}^{k, n}} .
\end{aligned}
$$

As in the estimate above for $C_{i j}^{n}$, condition (2.6) ensures that $\bar{a}_{i j}^{k, n}, \bar{b}_{i j}^{k, n} \in[0,1 / 3]$ and

$$
\sum_{i j} \tilde{C}_{i j}^{n} \leq \sum_{i j}\left|u_{i, j+1}^{k, n}-u_{i j}^{k, n}\right|
$$

by convexity. Passing to $\tilde{D}_{i j}^{n}$, we have:

$$
\tilde{D}_{i j}^{n}=F_{i+1 / 2, j}^{k, n}\left(u_{i, j+1}^{k, n}, u_{i+1, j+1}^{k, n}\right)-F_{i+1 / 2, j+1}^{k, n}\left(u_{i, j+1}^{k, n}, u_{i+1, j+1}^{k, n}\right)
$$




$$
\begin{aligned}
& +F_{i-1 / 2, j+1}^{k, n}\left(u_{i-1, j}^{k, n}, u_{i j}^{k, n}\right)-F_{i-1 / 2, j}^{k, n}\left(u_{i-1, j}^{k, n}, u_{i j}^{k, n}\right) \\
= & \frac{1}{2}\left(f_{i+1 / 2, j}^{k, n}\left(u_{i, j+1}^{k, n}\right)-f_{i+1 / 2, j+1}^{k, n}\left(u_{i, j+1}^{k, n}\right)+f_{i+1 / 2, j}^{k, n}\left(u_{i+1, j+1}^{k, n}\right)-f_{i+1 / 2, j+1}^{k, n}\left(u_{i+1, j+1}^{k, n}\right)\right. \\
& \left.-f_{i-1 / 2, j}^{k, n}\left(u_{i-1, j}^{k, n}\right)+f_{i-1 / 2, j+1}^{k, n}\left(u_{i-1, j}^{k, n}\right)-f_{i-1 / 2, j}^{k, n}\left(u_{i, j}^{k, n}\right)+f_{i-1 / 2, j+1}^{k, n}\left(u_{i, j}^{k, n}\right)\right) \\
= & \frac{1}{2}\left[f_{i+1 / 2, j}^{k, n}\left(u_{i, j+1}^{k, n}\right)-f_{i-1 / 2, j}^{k, n}\left(u_{i-1, j}^{k, n}\right)-\left[f_{i+1 / 2, j+1}^{k, n}\left(u_{i, j+1}^{k, n}\right)-f_{i-1 / 2, j+1}^{k, n}\left(u_{i-1, j}^{k, n}\right)\right](4.15)\right. \\
& \left.+f_{i+1 / 2, j}^{k, n}\left(u_{i+1, j+1}^{k, n}\right)-f_{i-1 / 2, j}^{k, n}\left(u_{i, j}^{k, n}\right)-f_{i+1 / 2, j+1}^{k, n}\left(u_{i+1, j+1}^{k, n}\right)+f_{i-1 / 2, j+1}^{k, n}\left(u_{i, j}^{k, n}\right)\right](4.16)
\end{aligned}
$$

We first estimate (4.15) using Lagrange's mean value theorem.

$$
\begin{aligned}
& =\frac{1}{2}\left(f^{k}\left(t^{n}, x_{i+1 / 2}, y_{j}, u_{i, j+1}^{k, n}, A_{i+1 / 2, j}^{n}\right)-f^{k}\left(t^{n}, x_{i-1 / 2}, y_{j}, u_{i-1, j}^{k, n}, A_{i-1 / 2, j}^{n}\right)\right) \\
& -\frac{1}{2}\left[f^{k}\left(t^{n}, x_{i+1 / 2}, y_{j+1}, u_{i, j+1}^{k, n}, A_{i+1 / 2, j+1}^{n}\right)-f^{k}\left(t^{n}, x_{i-1 / 2}, y_{j+1}, u_{i-1, j}^{k, n}, A_{i-1 / 2, j+1}^{n}\right)\right] \\
& =\frac{\Delta y}{2}\left(\partial_{y} f^{k}\left(t^{n}, x_{i-1 / 2}, \bar{y}_{j+1 / 2}, u_{i-1, j}^{k, n}, \bar{A}_{i-1 / 2, j+1 / 2}^{n}\right)\right. \\
& \left.\quad \quad-\partial_{y} f^{k}\left(t^{n}, x_{i+1 / 2}, \bar{y}_{j+1 / 2}, u_{i, j+1}^{k, n}, \bar{A}_{i+1 / 2, j+1 / 2}^{n}\right)\right) \\
& +\frac{1}{2} \nabla_{A} f^{k}\left(t^{n}, x_{i+1 / 2}, \bar{y}_{j+1 / 2}, u_{i, j+1}^{k, n}, \bar{A}_{i+1 / 2, j+1 / 2}^{n}\right)\left(A_{i+1 / 2, j}^{n}-A_{i+1 / 2, j+1}^{n}\right) \\
& -\frac{1}{2} \nabla_{A} f^{k}\left(t^{n}, x_{i-1 / 2}, \bar{y}_{j+1 / 2}, u_{i-1, j}^{k, n}, \bar{A}_{i-1 / 2, j+1 / 2}^{n}\right)\left(A_{i-1 / 2, j}^{n}-A_{i-1 / 2, j+1}^{n}\right)
\end{aligned}
$$

for suitable $\left.\bar{y}_{j+1 / 2} \in\right] y_{j}, y_{j+1}\left[\right.$ and $\bar{A}_{i-1 / 2, j+1 / 2}^{n} \in I\left(A_{i-1 / 2, j}^{n}, A_{i-1 / 2, j+1}^{n}\right)$. Introducing suitable $\left.\tilde{x}_{i}, \breve{x}_{i} \in\right] x_{i-1 / 2}, x_{i+1 / 2}\left[; \bar{u}_{i-1 / 2, j+1 / 2}^{k, n}, \check{u}_{i-1 / 2, j+1 / 2}^{k, n} \in I\left(u_{i-1, j}^{k, n}, u_{i+1, j+1}^{k, n}\right)\right.$; $\hat{A}_{i, j+1 / 2}^{n}, \check{A}_{i, j+1 / 2}^{n} \in I\left(\bar{A}_{i-1 / 2, j+1 / 2}^{n}, \bar{A}_{i+1 / 2, j+1 / 2}^{n}\right)$ and $\tilde{A}_{i j}^{n} \in I\left(A_{i-1 / 2, j}^{n}-A_{i-1 / 2, j+1}^{n}\right.$, $\left.A_{i+1 / 2, j}^{n}-A_{i+1 / 2, j+1}^{n}\right)$, we have

$$
\begin{aligned}
& \leq \frac{\Delta y}{2}\left(\Delta x\left|\partial_{x y}^{2} f^{k}\left(t^{n}, \tilde{x}_{i}, \bar{y}_{j+1 / 2}, \bar{u}_{i-1 / 2, j+1 / 2}^{k, n}, \hat{A}_{i, j+1 / 2}^{n}\right)\right|\right. \\
& +\left|\partial_{y} \partial_{u} f^{k}\left(t^{n}, \tilde{x}_{i}, \bar{y}_{j+1 / 2}, \bar{u}_{i-1 / 2, j+1 / 2}^{k, n}, \hat{A}_{i, j+1 / 2}^{n}\right)\right|\left|u_{i, j+1}^{k, n}-u_{i-1, j}^{k, n}\right|
\end{aligned}
$$$$
\left.+\left\|\partial_{y} \nabla_{A} f^{k}\left(t^{n}, \tilde{x}_{i}, \bar{y}_{j+1 / 2}, \bar{u}_{i-1 / 2, j+1 / 2}^{k, n}, \hat{A}_{i, j+1 / 2}^{n}\right)\right\|\left\|\bar{A}_{i+1 / 2, j+1 / 2}^{n}-\bar{A}_{i-1 / 2, j+1 / 2}^{n}\right\|\right)
$$$$
+\frac{1}{2}\left(\Delta x\left\|\partial_{x} \nabla_{A} f^{k}\left(t^{n}, \breve{x}_{i}, \bar{y}_{j+1 / 2}, \check{u}_{i-1 / 2, j+1 / 2}^{k, n}, \check{A}_{i, j+1 / 2}^{n}\right)\right\|\left\|\tilde{A}_{i j}^{n}\right\|\right.
$$$$
+\| \partial_{u} \nabla_{A} f^{k}\left(t^{n}, \breve{x}_{i}, \bar{y}_{j+1 / 2}, \check{u}_{i-1 / 2, j+1 / 2}^{k, n}, \check{A}_{i, j+1 / 2}^{n}\right)|||| \tilde{A}_{i j}^{n}||\left|u_{i, j+1}^{k, n}-u_{i-1, j}^{k, n}\right|
$$$$
+\left\|\nabla_{A A}^{2} f^{k}\left(t^{n}, \breve{x}_{i}, \bar{y}_{j+1 / 2}, \check{u}_{i-1 / 2, j+1 / 2}^{k, n}, \check{A}_{i, j+1 / 2}^{n}\right)\right\|\left\|\tilde{A}_{i j}^{n}\right\|\left\|\bar{A}_{i+1 / 2, j+1 / 2}^{n}-\bar{A}_{i-1 / 2, j+1 / 2}^{n}\right\|
$$

$$
\begin{aligned}
& +\left\|\nabla_{A} f^{k}\left(t^{n}, \breve{x}_{i}, \bar{y}_{j+1 / 2}, \check{u}_{i-1 / 2, j+1 / 2}^{k, n}, \check{A}_{i, j+1 / 2}^{n}\right)\right\| \\
& \left.\quad \times\left\|A_{i+1 / 2, j}^{n}-A_{i+1 / 2, j+1}^{n}-A_{i-1 / 2, j}^{n}+A_{i-1 / 2, j+1}^{n}\right\|\right) .
\end{aligned}
$$

We now use Lemma 4.2 since it holds that $\tilde{A}_{i j}^{n} \in I\left(A_{i-1 / 2, j}^{n}-A_{i-1 / 2, j+1}^{n}, A_{i+1 / 2, j}^{n}-\right.$ 
$\left.A_{i+1 / 2, j+1}^{n}\right)$ and $\bar{A}_{i-1 / 2, j+1 / 2}^{n} \in I\left(A_{i-1 / 2, j}^{n}, A_{i-1 / 2, j+1}^{n}\right)$, which implies that

$$
\begin{aligned}
\left\|\tilde{A}_{i j}^{n}\right\| & \leq \Delta y\left\|\partial_{y} \eta\right\|_{\mathbf{L}^{\infty}}\left\|u_{\Delta}\left(t^{n}\right)\right\|_{\mathbf{L}^{1}}, \\
\left\|\bar{A}_{i+1 / 2, j+1 / 2}^{n}-\bar{A}_{i-1 / 2, j+1 / 2}^{n}\right\| & \leq \Delta x\left\|\partial_{x} \eta\right\|_{\mathbf{L}^{\infty}}\left\|u_{\Delta}\left(t^{n}\right)\right\|_{\mathbf{L}^{1}}, \\
\left\|A_{i+1 / 2, j}^{n}-A_{i+1 / 2, j+1}^{n}-A_{i-1 / 2, j}^{n}+A_{i-1 / 2, j+1}^{n}\right\| & \leq \Delta x \Delta y\left\|\partial_{x y}^{2} \eta\right\|_{\mathbf{L}^{\infty}}\left\|u_{\Delta}\left(t^{n}\right)\right\|_{\mathbf{L}^{1}} .
\end{aligned}
$$

Therefore

$$
\begin{aligned}
2|(4.15)| \leq & M \Delta x \Delta y\left|\bar{u}_{i-1 / 2, j+1 / 2}^{k, n}\right|+\Delta y\left\|\partial_{y} \partial_{u} f^{k}\right\|_{\mathbf{L}^{\infty}}\left|u_{i, j+1}^{k, n}-u_{i-1, j}^{k, n}\right| \\
& +M \Delta x \Delta y\left|\bar{u}_{i-1 / 2, j+1 / 2}^{k, n}\right|\left\|\partial_{x} \eta\right\|_{\mathbf{L}^{\infty}}\left\|U_{o}\right\|_{\mathbf{L}^{1}} \\
& +M \Delta x \Delta y\left|\check{u}_{i-1 / 2, j+1 / 2}^{k, n}\right|\left\|\partial_{x} \eta\right\|_{\mathbf{L}^{\infty}}\left\|U_{o}\right\|_{\mathbf{L}^{1}} \\
& +\Delta y\left\|\partial_{u} \nabla_{A} f\right\|_{\mathbf{L}^{\infty}}\left\|\partial_{y} \eta\right\|_{\mathbf{L}^{\infty}}\left\|U_{o}\right\|_{\mathbf{L}^{1}}\left|u_{i, j+1}^{k, n}-u_{i-1, j}^{k, n}\right| \\
& +M \Delta x \Delta y\left|\check{u}_{i-1 / 2, j+1 / 2}^{k, n}\right|\left\|\partial_{x} \eta\right\|_{\mathbf{L}^{\infty}}\left\|\partial_{y} \eta\right\|_{\mathbf{L}^{\infty}}\left\|U_{o}\right\|_{\mathbf{L}^{1}}^{2} \\
& +M \Delta x \Delta y\left|\check{u}_{i-1 / 2, j+1 / 2}^{k, n}\right|\left\|\partial_{x y}^{2} \eta\right\|_{\mathbf{L}^{\infty}}\left\|U_{o}\right\|_{\mathbf{L}^{1}} \\
= & M \Delta x \Delta y\left|\bar{u}_{i-1 / 2, j+1 / 2}^{k, n}\right|\left(1+\left\|\partial_{x} \eta\right\|_{\mathbf{L}^{\infty}}\left\|U_{o}\right\|_{\mathbf{L}^{1}}\right) \\
& +M \Delta x \Delta y\left|\check{u}_{i-1 / 2, j+1 / 2}^{k, n}\right| \\
& \times\left(\left\|\partial_{x} \eta\right\|\left\|_{\mathbf{L}^{\infty}}\right\| \partial_{y} \eta\left\|_{\mathbf{L}^{\infty}}\right\| U_{o}\left\|_{\mathbf{L}^{1}}^{2}+\right\| \partial_{x y}^{2} \eta\left\|_{\mathbf{L}^{\infty}}\right\| U_{o}\left\|_{\mathbf{L}^{1}}+\right\| \partial_{x} \eta\left\|_{\mathbf{L}^{\infty}}\right\| U_{o} \|_{\mathbf{L}^{1}}\right) \\
& +\Delta y\left|u_{i, j+1}^{k, n}-u_{i-1, j}^{k, n}\right|\left(\left\|\partial_{u} \nabla_{A} f\right\|_{\mathbf{L}^{\infty}}\left\|\partial_{y} \eta\right\|_{\mathbf{L}^{\infty}}\left\|U_{o}\right\|_{\mathbf{L}^{1}}+\left\|\partial_{y} \partial_{u} f\right\|_{\mathbf{L}^{\infty}}\right),
\end{aligned}
$$

which implies that

$$
\sum_{i j}|(4.15)| \leq K_{2}+K_{3} \Delta y \sum_{i j}\left|u_{i, j+1}^{k, n}-u_{i-1, j}^{k, n}\right|
$$

where

$$
\begin{aligned}
& K_{2}=\frac{M}{2}\left\|U_{o}\right\|_{\mathbf{L}^{1}}\left[1+\left\|\partial_{x} \eta\right\|_{\mathbf{L}^{\infty}}\left\|U_{o}\right\|_{\mathbf{L}^{1}}\left(2+\left\|\partial_{y} \eta\right\|_{\mathbf{L}^{\infty}}\left\|U_{o}\right\|_{\mathbf{L}^{1}}\right)+\left\|\partial_{x y}^{2} \eta\right\|_{\mathbf{L}^{\infty}}\left\|U_{o}\right\|_{\mathbf{L}^{1}}\right] \\
& K_{3}=\frac{1}{2}\left(\left\|\partial_{u} \nabla_{A} f\right\|_{\mathbf{L}^{\infty}}\left\|\partial_{y} \eta\right\|_{\mathbf{L}^{\infty}}\left\|U_{o}\right\|_{\mathbf{L}^{1}}+\left\|\partial_{y} \partial_{u} f\right\|_{\mathbf{L}^{\infty}}\right) .
\end{aligned}
$$

Similarly,

$$
\sum_{i j}|(4.16)| \leq K_{2}+K_{3} \Delta y \sum_{i j}\left|u_{i+1, j+1}^{k, n}-u_{i j}^{k, n}\right|
$$

Therefore

$$
\begin{aligned}
\sum_{i j} \lambda_{x}\left|\tilde{D}_{i j}^{n}\right| \Delta x & \leq \sum_{i j} \lambda_{x}(|(4.15)|+|(4.16)|) \Delta x \\
& \leq 2 K_{2} \Delta t+K_{3} r \Delta t \sum_{i j}\left(\left|u_{i+1, j}^{k, n}-u_{i j}^{k, n}\right| \Delta y+\left|u_{i, j+1}^{k, n}-u_{i j}^{k, n}\right| \Delta x\right)
\end{aligned}
$$

where

$$
r=\max \left\{\frac{\Delta x}{\Delta y}, \frac{\Delta y}{\Delta x}\right\} .
$$


Collecting the various terms above, we get

$$
\begin{aligned}
\sum_{i j}\left|u_{i, j+1}^{k, n+1 / 2}-u_{i j}^{k, n+1 / 2}\right| \Delta x \leq & \left(1+K_{3} r \Delta t\right) \sum_{i j}\left|u_{i, j+1}^{k, n}-u_{i j}^{k, n}\right| \Delta x \\
& +K_{3} r \Delta t \sum_{i j}\left|u_{i+1, j}^{k, n}-u_{i j}^{k, n}\right| \Delta y+K_{2} \Delta t,
\end{aligned}
$$

and, finally,

$$
\begin{aligned}
& \sum_{i j}\left(\left|u_{i+1, j}^{k, n+1 / 2}-u_{i j}^{k, n+1 / 2}\right| \Delta y+\left|u_{i, j+1}^{k, n+1 / 2}-u_{i j}^{k, n+1 / 2}\right| \Delta x\right) \\
\leq & \left(1+\mathcal{K}_{1} \Delta t\right) \sum_{i j}\left(\left|u_{i+1, j}^{k, n}-u_{i j}^{k, n}\right| \Delta y+\left|u_{i, j+1}^{k, n}-u_{i j}^{k, n}\right| \Delta x\right)+\mathcal{K}_{2} \Delta t,
\end{aligned}
$$

where, using the definitions (4.13), (4.17) and (4.18),

$$
\mathcal{K}_{1}=K_{1}+K_{3} r \quad \text { and } \quad \mathcal{K}_{2}=\frac{K+K_{2}}{K_{1}+K_{3} r} .
$$

Entirely analogous estimates lead to

$$
\begin{aligned}
& \sum_{i j}\left(\left|u_{i+1, j}^{k, n+1}-u_{i j}^{k, n+1}\right| \Delta y+\left|u_{i, j+1}^{k, n+1}-u_{i j}^{k, n+1}\right| \Delta x\right) \\
\leq & \left(1+\mathcal{K}_{1} \Delta t\right) \sum_{i j}\left(\left|u_{i+1, j}^{k, n+1 / 2}-u_{i j}^{k, n+1 / 2}\right| \Delta y+\left|u_{i, j+1}^{k, n+1 / 2}-u_{i j}^{k, n+1 / 2}\right| \Delta x\right)+\mathcal{K}_{1} \mathcal{K}_{2} \Delta t,
\end{aligned}
$$

so that, recursively,

$$
\begin{aligned}
& \sum_{i j}\left(\left|u_{i+1, j}^{k, n}-u_{i j}^{k, n}\right| \Delta y+\left|u_{i, j+1}^{k, n}-u_{i j}^{k, n}\right| \Delta x\right) \\
\leq & e^{2 \mathcal{K}_{1} t^{n}} \sum_{i j}\left(\left|u_{i+1, j}^{k, 0}-u_{i j}^{k, 0}\right| \Delta y+\left|u_{i, j+1}^{k, 0}-u_{i j}^{k, 0}\right| \Delta x\right)+\mathcal{K}_{2}\left(e^{2 \mathcal{K}_{1} t^{n}}-1\right)
\end{aligned}
$$

completing the proof of the lemma.

Proof of Lemma 2.8. Following [3, Lemma 2.7] and [14, Section 3], we have

$$
\begin{aligned}
& \sum_{i j}\left|u_{i j}^{k, n+1 / 2}-u_{i j}^{k, n}\right| \Delta x \Delta y \\
\leq & \frac{\Delta t}{2} \Delta y \sum_{i j}\left|f^{k}\left(t^{n}, x_{i+1 / 2}, y_{j}, u_{i j}^{k, n}, A_{i+1 / 2, j}^{n}\right)-f^{k}\left(t^{n}, x_{i-1 / 2}, y_{j}, u_{i-1, j}^{k, n}, A_{i-1 / 2, j}^{n}\right)\right|(4.20) \\
+ & \frac{\Delta t}{2} \Delta y \sum_{i j}\left|f^{k}\left(t^{n}, x_{i+1 / 2}, y_{j}, u_{i+1, j}^{k, n}, A_{i+1 / 2, j}^{n}\right)-f^{k}\left(t^{n}, x_{i-1 / 2}, y_{j}, u_{i j}^{k, n}, A_{i-1 / 2, j}^{n}\right)\right|(4.21) \\
& +\alpha \sum_{i j} \Delta x \Delta y\left|u_{i+1, j}^{k, n}-u_{i j}^{k, n}\right| .
\end{aligned}
$$

Consider (4.20), since (4.21) can be treated in the same way. Using Lemma 4.2

$$
(4.20)=\frac{\Delta t}{2} \Delta y \sum_{i j}\left|f^{k}\left(t^{n}, x_{i+1 / 2}, y_{j}, u_{i j}^{k, n}, A_{i+1 / 2, j}^{n}\right)-f^{k}\left(t^{n}, x_{i-1 / 2}, y_{j}, u_{i-1, j}^{k, n}, A_{i-1 / 2, j}^{n}\right)\right|
$$




$$
\begin{aligned}
& \leq \frac{\Delta t}{2} \Delta y \sum_{i j}\left[\left|\partial_{x} f^{k}\left(t^{n}, \bar{x}_{i}, y_{j}, \bar{u}_{i-1 / 2, j}^{k, n}, \bar{A}_{i j}^{n}\right)\right| \Delta x\right. \\
& +\left|\partial_{u} f^{k}\left(t^{n}, \bar{x}_{i}, y_{j}, \bar{u}_{i-1 / 2, j}^{k, n}, \bar{A}_{i j}^{n}\right)\right|\left|u_{i j}^{k, n}-u_{i-1, j}^{k, n}\right| \\
& \left.+\left\|\nabla_{A} f\left(t^{n}, \bar{x}_{i}, y_{j}, \bar{u}_{i-1 / 2, j}^{k, n}, \bar{A}_{i j}^{n}\right)\right\|\left\|A_{i+1 / 2, j}^{n}-A_{i-1 / 2, j}^{n}\right\|\right] \\
& \leq \frac{\Delta t}{2} \Delta y \sum_{i j}\left[M \Delta x\left|\bar{u}_{i-1 / 2, j}^{k, n}\right|\left(1+\left\|\partial_{x} \eta\right\|_{\mathbf{L}^{\infty}}\left\|U_{o}\right\|_{\mathbf{L}^{1}}\right)+\left\|\partial_{u} f\right\|_{\mathbf{L}^{\infty}}\left|u_{i j}^{k, n}-u_{i-1, j}^{k, n}\right|\right] \\
& \leq M \frac{\Delta t}{2}\left\|U_{o}\right\|_{\mathbf{L}^{1}}\left(1+\left\|\partial_{x} \eta\right\|_{\mathbf{L}^{\infty}}\left\|U_{o}\right\|_{\mathbf{L}^{1}}\right)+\frac{\Delta t}{2}\left\|\partial_{u} f\right\|_{\mathbf{L}^{\infty}} \sum_{i j} \Delta y\left|u_{i+1, j}^{k, n}-u_{i j}^{k, n}\right|,
\end{aligned}
$$

so that

$$
\begin{aligned}
\sum_{i j}\left|u_{i j}^{k, n+1 / 2}-u_{i j}^{k, n}\right| \Delta x \Delta y \leq & M \Delta t\left\|U_{o}\right\|_{\mathbf{L}^{1}}\left(1+\left\|\partial_{x} \eta\right\|_{\mathbf{L}^{\infty}}\left\|U_{o}\right\|_{\mathbf{L}^{1}}\right) \\
& +\Delta t\left(\left\|\partial_{u} f\right\|_{\mathbf{L}^{\infty}}+\frac{\alpha}{\lambda_{x}}\right) \sum_{i j} \Delta y\left|u_{i+1, j}^{k, n}-u_{i j}^{k, n}\right|,
\end{aligned}
$$

which, together with the analogous estimate for $\sum_{i j}\left|u_{i j}^{n+1}-u_{i j}^{k, n+1 / 2}\right| \Delta x \Delta y$ completes the proof of the Lemma with

$$
\begin{aligned}
C= & \sqrt{n} M\left\|U_{o}\right\|_{\mathbf{L}^{1}}\left(2+\left(\left\|\partial_{x} \eta\right\|_{\mathbf{L}^{\infty}}+\left\|\partial_{y} \vartheta\right\|_{\mathbf{L}^{\infty}}\right)\left\|U_{o}\right\|_{\mathbf{L}^{1}}\right) \\
& +\sqrt{n}\left(\left\|\partial_{u} f\right\|_{\mathbf{L}^{\infty}}+\frac{\alpha}{\lambda_{x}}+\left\|\partial_{u} g\right\|_{\mathbf{L}^{\infty}}+\frac{\beta}{\lambda_{y}}\right)\left(e^{\mathcal{K}_{1} t^{n}} \operatorname{TV}\left(U_{o}\right)+\mathcal{K}_{2}\left(e^{\mathcal{K}_{1} t^{n}}-1\right)\right) .
\end{aligned}
$$

Proof of Theorem 2.2. The strong BV estimate proved in Lemma 2.6 allows to use classical tools. Indeed, for instance, all properties 1. to 6. in [20, Section 3] hold. More precisely, 1. follows from Lemma 2.4, 2. follows from Lemma 2.6 through [20, Lemma 3.3], 3. follows from Lemma 2.8, 4. follows from Lemma 2.5, 5 . follows from 6 ., and 6. follows from Lemma 2.7. Hence, for any sequence $(\Delta t)_{\nu},(\Delta x)_{\nu},(\Delta y)_{\nu}$ tending to 0 and satisfying for any $\nu$ the CFL condition (2.6), an application of [20, Proposition 3.1] ensures the convergence (up to a subsequence) of the approximate solutions $u_{\Delta_{\nu}}^{k}$ to a solution $U^{k}$ of $(2.2)$, for $k=1, \ldots, N$.

By (2.4)-(2.5), the terms $A$ and $B$ converge to the respective convolutions integrals, ensuring that $U^{k}$ solves (1.1) in the sense of Definition 2.1.

Finally, the uniformity of the bounds proved in the Lemmas allows to pass each of these bounds to the solution, completing the proof.

Proof of Lemma 3.1. It is sufficient to verify that (H0), (H1), (H2) and (H3) hold. The latter condition is immediate. Clearly, $f$ and $g$ as defined in (3.2) are both of class $\mathbf{C}^{2}$ in all their arguments.

Let now $p \in \mathbf{C}_{\mathbf{c}}^{\mathbf{3}}(\mathbb{R} ; \mathbb{R})$ be such that $p(U)=U(1-U)$ for all $U \in[0,1]$ and $q \in \mathbf{C}_{\mathbf{c}}^{\mathbf{3}}(\mathbb{R} ; \mathbb{R})$ be such that $q(A)=1-A$ for all $A \in[0,1]$. Define

$$
\hat{f}(t, x, y, U, A)=p(U) q(A) v^{1}(x, y) \quad \text { and } \quad \hat{g}(t, x, y, U, B)=p(U) q(B) v^{2}(x, y) .
$$

Clearly, by the above choices and by (3.3), $\hat{f}$ and $\hat{g}$ both are of class $\mathbf{C}^{\mathbf{2}} \cap \mathbf{W}^{\mathbf{3}, \infty}$. 
Then, Theorem 2.2 applies and yields a solution to $\partial_{t} U+\nabla \cdot(p(U) q(U * \mu) v)=0$ in the sense of Definition 2.1. By [17, Theorem 3], $u$ attains values inside $[0,1]$, hence it also solves (3.1).

Acknowledgment: This work was partially supported by the European Research Council under the European Union's Seventh Framework Program (FP/2007-2013) ERC Grant Agreement n. 257661. The second author was partially supported by the INDAM-GNAMPA project Conservation Laws: Theory and Applications.

\section{REFERENCES}

[1] Debora Amadori and Wen Shen, An integro-differential conservation law arising in a model of granular flow, J. Hyperbolic Differ. Equ., 9 (2012), pp. 105-131.

[2] Luigi Ambrosio, François Bouchut, and Camillo De Lellis, Well-posedness for a class of hyperbolic systems of conservation laws in several space dimensions, Comm. Partial Differential Equations, 29 (2004), pp. 1635-1651.

[3] Paulo Amorim, Rinaldo M. Colombo, and Andreia Teixeira, On the numerical integration of scalar nonlocal conservation laws, Preprint, (2013).

[4] F. Betancourt, R. Bürger, K. H. Karlsen, and E. M. Tory, On nonlocal conservation laws modelling sedimentation, Nonlinearity, 24 (2011), pp. 855-885.

[5] Sebasten Blandin and Paola Goatin, Well-posedness of a conservation law with non-local flux arising in traffic flow modeling, Preprint, (2014).

[6] Raul Borsche, Rinaldo M. Colombo, Mauro Garavello, and Anne Meurer, Differential equations modeling crowd interactions, Preprint, (2014).

[7] Claire Chainais-Hillairet, Finite volume schemes for a nonlinear hyperbolic equation. Convergence towards the entropy solution and error estimate, M2AN Math. Model. Numer. Anal., 33 (1999), pp. 129-156.

[8] Rinaldo M. Colombo, Mauro Garavello, and Magali Lécureux-Mercier, A class of nonlocal models for pedestrian traffic, Math. Models Methods Appl. Sci., 22 (2012), pp. 1150023,34 .

[9] Rinaldo M. Colombo, Michael Herty, and Magali Mercier, Control of the continuity equation with a non local flow, ESAIM Control Optim. Calc. Var., 17 (2011), pp. 353-379.

[10] Rinaldo M. Colombo and Lécureux-Magali Mercier, Nonlocal crowd dynamics models for several populations, Acta Mathematica Scientia, 32 (2011), pp. 177-196.

[11] Michael Crandall and Andrew Majda, The method of fractional steps for conservation laws, Numer. Math., 34 (1980), pp. 285-314.

[12] Michael G. Crandall and Andrew Majda, Monotone difference approximations for scalar conservation laws, Math. Comp., 34 (1980), pp. 1-21.

[13] Simone Göttlich, Simon Hoher, Patrick Schindler, Veronika Schleper, and Alexander VERL, Modeling, simulation and validation of material flow on conveyor belts, Appl. Math. Modell., to appear (2013), p. 29 pages.

[14] Kenneth Hvistendahl Karlsen and Nils Henrik Risebro, Convergence of finite difference schemes for viscous and inviscid conservation laws with rough coefficients, M2AN Math. Model. Numer. Anal., 35 (2001), pp. 239-269.

[15] Barbara L. Keyfitz and Herbert C. Kranzer, A system of nonstrictly hyperbolic conservation laws arising in elasticity theory, Arch. Rational Mech. Anal., 72 (1979/80), pp. 219241.

[16] Ujuwal Koley and Nils Henrik Risebro, Finite difference schemes for the symmetric keyfitzkranzer system, Zeitschrift für angewandte Mathematik und Physik, 64 (2013), pp. 10571085.

[17] Stanislav Nikolaevich KružHKov, First order quasilinear equations with several independent variables., Mat. Sb. (N.S.), 81 (123) (1970), pp. 228-255.

[18] Randall J. LeVeque, Finite volume methods for hyperbolic problems, Cambridge Texts in Applied Mathematics, Cambridge University Press, Cambridge, 2002.

[19] Benoît Perthame, Transport equations in biology, Frontiers in Mathematics, Birkhäuser Verlag, Basel, 2007.

[20] Richard Sanders, On convergence of monotone finite difference schemes with variable spatial differencing, Math. Comp., 40 (1983), pp. 91-106. 